MATHEMATICS OF COMPUTATION

Volume 75, Number 256, October 2006, Pages 1779-1808

S 0025-5718(06)01924-7

Article electronically published on June 14, 2006

\title{
JACOBI'S LAST GEOMETRIC STATEMENT EXTENDS TO A WIDER CLASS OF LIOUVILLE SURFACES
}

\author{
ROBERT SINCLAIR AND MINORU TANAKA
}

\begin{abstract}
Numerical evidence is presented which strongly suggests that "Jacobi's last geometric statement"- that the conjugate locus from a point has exactly four cusps and the corresponding cut locus consists of only one topological segment - holds for compact real analytic Liouville surfaces diffeomorphic to $S^{2}$ if the Gaussian curvature is everywhere positive and has exactly six critical points, these being two saddles, two global minima, and two global maxima (as is the case for an ellipsoid). Our experiments suggest that this is a sufficient rather than a necessary condition. Furthermore, for compact real analytic Liouville surfaces diffeomorphic to $S^{2}$ upon which the Gaussian curvature can be negative but has exactly six critical points, these being two saddles, two global minima, and two global maxima, it appears that the cut locus is always a subarc of a line given by $x_{1}=$ const or $x_{2}=$ const, where $\left(x_{1}, x_{2}\right)$ are canonical coordinates with respect to which the metric has the form $\left(f_{1}\left(x_{1}\right)+f_{2}\left(x_{2}\right)\right)\left(d x_{1}^{2}+d x_{2}^{2}\right)$. In the case of an ellipsoid, these curves are lines of curvature.
\end{abstract}

The point of this paper is to present a conjecture, already contained in the abstract, as a contribution to pure mathematical research in global Riemannian geometry. The overwhelming bulk of the paper will however necessarily be devoted to a description of the computational methods used to motivate the conjecture, and, in particular, the checks which have been performed to verify that the software (which has been written specifically for this study) is indeed performing correctly. Such checks are vital in any experimental work. This paper is not intended to be a contribution to computational science as such, since the algorithms we have used, although perhaps combined in an unusual manner, are standard.

The conjugate locus from a point $p$ (which we will often refer to as the starting point) on a Riemannian manifold is the envelope of the geodesics emanating from $p$. In two dimensions, it can also be defined analogously to the nodes of a vibrating string as follows. A geodesic passing through $p$ can be uniquely defined by the angle $\theta$ it makes at $p$ with one fixed (reference) geodesic also passing through $p$. Varying this angle by a small amount $(\delta \theta)$ will cause the geodesic to deviate from its original path. The distance between points on the geodesic with angle $\theta$ (say $g_{\theta}$ ) and the geodesic curve with angle $\theta+\delta \theta$ (write $g_{\theta+\delta \theta}$ ) can be written as a real function $\xi_{\delta \theta}$ of arclength $s$ along $g_{\theta}$, where $s=0$ corresponds to the point $p$. Clearly $\xi_{\delta \theta}(0)=0$, since we are only considering geodesics which pass through $p$. The limit of $\xi_{\delta \theta}(s) / \delta \theta$ as $\delta \theta$ approaches zero may have further zeros (the nodes of the string, where, to

Received by the editor October 4, 2004.

2000 Mathematics Subject Classification. Primary 53C20, 53-04; Secondary 53C25.

Key words and phrases. Cut locus, conjugate locus, Liouville surface.

(C)2006 American Mathematical Society Reverts to public domain 28 years from publication 
first order, the string does not move when plucked). The points at which these zeros appear are said to be conjugate to $p$. In physics, one refers to the distance $\xi$ as the "geodesic deviation" (Section 1.6 of [42]). See Section 2 of Chapter II of [54] for a precise definition of conjugate points, where the theory of Jacobi fields generalizes the simple notion of geodesic deviation as introduced above to dimensions greater than two (see also Chapter 11 of [42]). From the point of view of geometrical optics, one can imagine the set of focal points of rays emanating from the starting point $p$ (see, for example, Figure 8.1 of [22]).

The cut locus from a point $p$ on a complete Riemannian manifold is the closure of the set of points $q$ which have at least two minimal geodesic connections to $p$ 64. Again, see Section 4 of Chapter III of [54 for more details. In a rough sense, the cut locus corresponds to the boundary past which no path from the starting point can be minimizing.

What is known as "Jacobi's last geometric statement" originated in a discussion of the principle of least action in the sixth of Jacobi's Lectures on Dynamics [27, delivered in the winter of $1842 / 1843$. The example he used was an ellipsoid of revolution. He stated without proof that the conjugate locus from a general point has exactly four cusps, from which it would follow that the cut locus is a single topological segment with two endpoints. Despite the importance of this result (see Chapter 3 of [2, Section TOP 4 of [6], also 60] and references therein), it has only recently been proven that the cut locus from a general point of an ellipsoid of revolution does indeed have only two endpoints 61. Actually, Jacobi's last geometric statement is commonly thought to have concerned general ellipsoids. This case was also only dealt with recently [25. In both cases, computational experimentation [26, 60] provided some insights which were useful in the construction of the pure mathematical proofs. We sincerely hope that this paper will also inspire further mathematical proofs.

Jacobi integrated the geodesic flow on triaxial ellipsoids using a remarkable variable substitution. Liouville was able to integrate the geodesic flow of a wider class of surfaces (Liouville surfaces; see the references given below) containing the triaxial ellipsoids as special cases. The starting point of this investigation is the obvious question as to whether (or, if so, how far) Jacobi's statement applies to Liouville surfaces which are not ellipsoids.

We have heard that Professors Jin-Ichi Itoh of Kumamoto University and Kazuyoshi Kiyohara of Okayama University (the authors of [25]) have, working independently from us, already been able to prove theorems of a similar nature to our conjecture, but for a class of Liouville surfaces defined differently from ours (and, we believe, different from the class we consider despite the fact that there must be some overlap). Their work is as yet unpublished.

We will consider only simply connected, compact, complete two-dimensional real analytic Riemannian surfaces. Their conjugate and cut loci were first investigated by Poincaré 48, and Myers [43, 44.

Real analytic Liouville surfaces have a Riemannian metric locally of the form

$$
d s^{2}=\left[f_{1}\left(x_{1}\right)+f_{2}\left(x_{2}\right)\right]\left(d x_{1}^{2}+d x_{2}^{2}\right),
$$

which can also be expressed as

$$
d s^{2}=(U-V)\left(U_{1}^{2} d u^{2}+V_{1}^{2} d v^{2}\right),
$$


where $U$ and $U_{1}$ are functions of $u$ only, and $V$ and $V_{1}$ of $v$ only (see Chapter I of Book VI of [16, originally published in 1894). An example of such surfaces are the triaxial ellipsoids. Their geodesic flows were first shown to be integrable by Jacobi in the 28th of his Lectures on Dynamics [27. Liouville was able to generalize this result to the class of surfaces which bear his name (see Chapter XIV of Book IV of [15, first published in 1915, and Chapters I and II of Book VI of [16]).

We will call the coordinates $\left(x_{1}, x_{2}\right)$ with respect to which the metric assumes the form of equation (0.1) canonical coordinates.

Liouville surfaces 32, 23, Kähler-Liouville manifolds 33, 24, and surfaces of revolution (see Sections 2-2 and 4-2 of [62] and Chapter 7 of [58]) are the most general classes of surfaces with linear or quadratic integrable geodesic flow (i.e. the integrals of the geodesic flows are linear or quadratic with respect to the momenta [10, 11]).

The computational method we will use to approximate the cut locus is a hybrid approach, in which we actually approximate both the cut and conjugate loci, making use of the fact that the endpoints of the cut locus are cusps of the conjugate locus turned towards the starting point (called "foyers en pointe" in [4]).

We approximate the cut locus from a point $p$ by first computing approximations to the distance function $d(p, \cdot)$ on a regular grid using a level set approach [45, 31, 57. and then applying a feature extraction operator based upon the Laplacian to these values. The theoretical basis of this approach is that the set of limit vectors of the gradient vector field of the distance function from $p$ has at least two vectors at any normal cut point of a point $p$ on a Riemannian manifold of any number of dimensions. Of course we consider the gradient vector field of the distance function to be defined on the outside of the cut locus (and excepting the starting point). The distance function is perhaps one of the most basic objects of global Riemannian geometry, having deep connections with the heat kernel via Varadhan's result [40, and topology via Morse theory [12, 21. It is also of practical relevance in, for example, vehicle path planning [63]. Chapters 6 and 7 of [31] describe some applications and contain a particularly clear introduction to the methods used. 29] is a useful early reference in this field. Our computational approach borrows heavily from this body of knowledge.

The cut locus is intimitely related to Voronoi diagrams [3, 52, 35], skeletons (Section 6.4 of [31] and [66]), and the medial axis [9, 65] of bounded domains. See [13, 14] and the references therein for a discussion of further related constructions. The Voronoi diagram of a set of sites $S$ is a subdivision of space into cells, each consisting of the points closest to a particular site. The medial axis of a closed bounded domain in $\mathbb{E}^{2}$, as it is typically defined, can be intuitively thought of as the closure of the locus of centres of maximum-radius circles (touching the boundary in at least two points) which may be inscribed within the domain [50. One can also define the medial axis of a closed bounded domain as the set of all centres of maximal-radius balls in the domain. For example, consider the domain $D$ bounded by an ellipsoid $x^{2} / 4+y^{2}=1$. The medial axis of $D$ is $[-b, b] \times\{0\}$, where $b=1 / K$ with $K$ the curvature of the ellipsoid at the point $(2,0)$. The maximal-radius ball centred at $(-b, 0)$ is tangent to the ellipsoid at the "unique" point $(-2,0)$, but not at more than one point. It is of interest to note that it appears 37] that our eyes are capable of "computing" the medial axes of objects we see, as an aid to the higher-level recognition processes in our brains. On a compact surface, the 
(obvious generalization of the) medial axis of any geodesic circle of small enough radius centred at a point $p$ is the union of that point and that point's cut locus. See 65. for a more detailed discussion (and redefinition of) Blum's medial axis transform, Voronoi diagrams, and their relationship with the cut locus.

The main differences between our needs (in a pure mathematical context) and those of applied mathematicians are that (i) we can essentially disregard "noise" (although we must keep an eye on floating-point errors 19, but these are orders of magnitude smaller than the measurement errors typically met in real-world applications) and (ii) our primary aim is to make reliable statements concerning quantitative aspects of the cut locus, whereas an applied mathematician will more typically be concerned with computational complexity (speed and memory use) and interested in really useful algorithms which may only mirror qualitative aspects of the mathematical cut locus. See the introduction of [30] for a discussion of the latter style of approach.

\section{Computing the distance map to locate the Cut locus}

We wish to approximate the cut locus from a fixed point $p$. The distance from $p$ to any other point $q$ (i.e., $d(p, q))$ is a continuous function, but nondifferentiable at $q=p$ and (see below for details) for $q$ on a dense set of points of the cut locus of $p$.

Let the point $q$ have coordinates $\left(x_{1}, x_{2}\right)$. Then we can define the distance function

$$
t\left(x_{1}, x_{2}\right)=d\left(p,\left(x_{1}, x_{2}\right)\right) .
$$

Wherever it is differentiable, the distance function obeys

$$
\left\|\nabla t\left(x_{1}, x_{2}\right)\right\|_{2}=\sqrt{f_{1}\left(x_{1}\right)+f_{2}\left(x_{2}\right)}
$$

(as a consequence of the Gauss Lemma; see Section 3.2 of [47]), which is an Eikonal equation for an isotropic medium, a special case of the Hamilton-Jacobi equation. Such equations have been studied extensively in the context of acoustic wave propagation in geophysics, where what we have called the distance function is known as the traveltime, fluid flow, computer vision, semiconductor fabrication (etching and deposition processes), and many more areas. See [28, 46, 5, 53, 36] for discussions and comparisons of some of the methods used by applied mathematicians and geophysicists to compute traveltimes. Of these, the fast marching level set method [56] is a particularly efficient and robust algorithm.

We will work on a regular rectangular grid with distance $\Delta x_{1}$ between neighbouring points along the $x_{1}$ axis and $\Delta x_{2}$ along the $x_{2}$ axis. In all cases, the number of grid points in each direction will be chosen to ensure that $\Delta x_{1} \approx \Delta x_{2}$. Initially, we assign the distance zero to the grid point closest to the starting point $p$ (we are interested in investigating cut loci from generic rather than specific starting points). We can assign a distance from the starting point to a grid point with coordinates $\left(x_{1}, x_{2}\right)$ if at least one of this grid point's neighbours has already been assigned a distance. The following is an adaptation of the exposition in Section 2.3 of 28 to Liouville surfaces. If only one neighbour, say the one at $\left(x_{1}+\Delta x_{1}, x_{2}\right)$, has been assigned a distance $\left(t_{1}\right)$ to $p$, then we can use

$$
t_{0}=t_{1}+\Delta x_{1} \sqrt{f_{1}\left(x_{1}\right)+f_{2}\left(x_{2}\right)}
$$


to assign a distance $\left(t_{0}\right)$ to $p$ from this grid point. If two neighbours, one in the direction of the $x_{1}$ axis (forwards or backwards) and another in the direction of the $x_{2}$ axis (forwards or backwards), have been assigned distances to $p, t_{1}$ and $t_{2}$ respectively, then we can use

$$
t_{0}=\frac{t_{1} s_{2}+t_{2} s_{1}+\sqrt{\left(t_{1} s_{2}+t_{2} s_{1}\right)^{2}+\left(s_{1}+s_{2}\right)\left[s_{1} s_{2}\left(f_{1}\left(x_{1}\right)+f_{2}\left(x_{2}\right)\right)-t_{1}^{2} s_{2}-t_{2}^{2} s_{1}\right]}}{s_{1}+s_{2}}
$$

where we have written $s_{1}$ for $\left(\Delta x_{1}\right)^{2}$ and $s_{2}$ for $\left(\Delta x_{2}\right)^{2}$. This process can be repeated iteratively, using these two equations to update distances, always choosing the smallest distance from $p$ for any grid point. We did not implement the heapsort structure so vital to ensuring efficiency in the fast marching level set method [56] since we were more concerned with correctness than speed. In fact, we found our implementation to be fast enough for our purposes.

We are now faced with the task of feature-extraction. We know that the closure of the set of nonsmooth points of the distance function equals the cut locus of the starting point along with the starting point itself [8]. At the starting point $p=\left(x_{1}^{p}, x_{2}^{p}\right)$, we have

$$
t(x):=d(x, p) \approx \sqrt{f_{1}\left(x_{1}^{p}\right)+f_{2}\left(x_{2}^{p}\right)} \times\|x-p\|_{2} .
$$

At ordinary cut points of the cut locus (where there are two or more minimizing segments from $p$ ) there are two different values of the gradient of the distance function [8]. Also, the form of the distance function at a cut point is clearly that of a ridge rather than a valley. See Figure 1 for an example of a distance map. We are therefore dealing with what is known in the literature as a roof edge [38, but we are in the enviable position of being able to compute an almost noiseless approximation to the distance function. This means that we will not need the smoothing or fitting procedures so common in image processing. We will make use of the idea that a
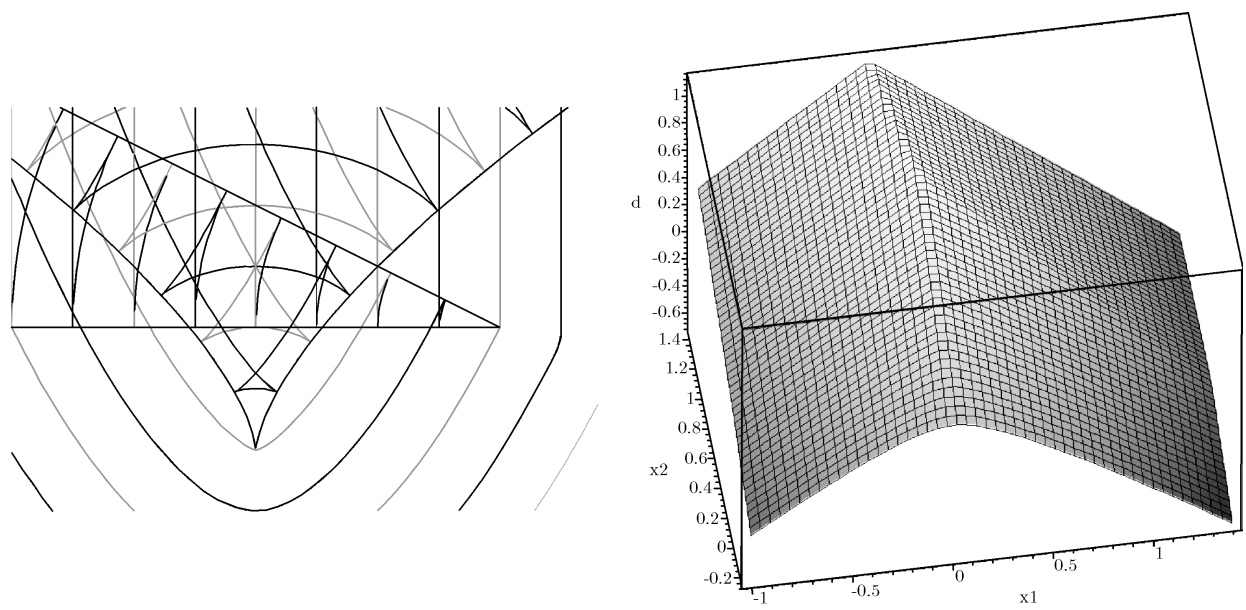

Figure 1. Wavefronts (alternating light/dark) moving in the plane with the corresponding caustic, shown on the left, and the distance map shown on the right, where distances are measured with respect to the wavefront at time $t=0$. 
roof edge can be located by using what is known as a Laplacian kernel. The idea is that at points where the distance function is smooth, the standard finite-difference approximation to the Laplacian on a regular square grid

$$
\nabla f \propto f_{i-1, j}+f_{i, j-1}-4 f_{i, j}+f_{i+1, j}+f_{i, j+1}
$$

(note that we have discarded the denominator) scales as the square of the distance between neighbouring grid points (the absolute value of the difference between corresponding $x_{1}$ or $x_{2}$ values), whereas it scales linearly with this distance at a roof edge point. In the limit of vanishing distance between grid points, values of the absolute value of the Lapacian kernel at ordinary cut points or the starting point itself will be infinitely greater than at any other points. In this limit, the cut locus should therefore be immediately recognizable.

Of course we will have to make do with finite distances between neighbouring points. We initially used

$$
L^{(1)}\left(x_{1}, x_{2}\right)=\left|L\left(x_{1}, x_{2}, d\left(\left(x_{1}^{p}, x_{2}^{p}\right),(\cdot, \cdot)\right)\right)\right|,
$$

where

$$
\begin{aligned}
L\left(x_{1}, x_{2}, t\right) & =t\left(x_{1}-\Delta x_{1}, x_{2}\right)-2 t\left(x_{1}, x_{2}\right)+t\left(x_{1}+\Delta x_{1}, x_{2}\right) \\
& +t\left(x_{1}, x_{2}-\Delta x_{2}\right)-2 t\left(x_{1}, x_{2}\right)+t\left(x_{1}, x_{2}+\Delta x_{2}\right) .
\end{aligned}
$$

We found that values of $L^{(1)}$ varied so much over the cut locus that it was difficult to construct a clear image from them, so we instead computed

$$
L^{(2)}\left(x_{1}, x_{2}\right)=\left|L\left(x_{1}, x_{2}, d\left(\left(x_{1}^{p}, x_{2}^{p}\right),(\cdot, \cdot)\right)\right)\right|^{1 / 4}
$$

at every grid point, scaled the values linearly to the range $[0,1]$, and then marked those points at which the scaled values were larger than some constant (we used 0.4 , but note that this constant can and should be lowered to zero as the number of grid points tends to infinity).

One can also work with the distance squared $d^{2}(p, q)$, which has the advantage that it is smooth at the starting point. For points $q$ joined by two minimal geodesics to the starting point $p$, which are dense in the cut locus of $p$, we know that $d^{2}(p, \cdot)$ has no directional derivative at $q$ for vectors in the direction of these two minimal geodesics [64. We have therefore also used

$$
L^{(3)}\left(x_{1}, x_{2}\right)=\log _{e}\left|L\left(x_{1}, x_{2}, d^{2}\left(\left(x_{1}^{p}, x_{2}^{p}\right),(\cdot, \cdot)\right)\right)\right|,
$$

mapping those grid points at which $L^{(3)}$ is at least 4 less than the maximum value over all grid points to the range $[0,1]$, and then shading these points (only) using their normalized values. Once again, this value of 4 is suited only to the typical grid sizes we have used. It can and should be increased as the number of grid points tends to infinity.

1.1. A generic model. The point of this section is to introduce an exactly solvable but also truly representative model which can be used to check our software.

We know [12] that the structure of a generic (stable) cut locus for compact twodimensional manifolds is such that near a point $q$ the cut locus with respect to $p$ has one of three forms:

(i) a straight line through $q$ or

(ii) a straight line starting at $q$ or

(iii) three straight lines meeting at $q$. 

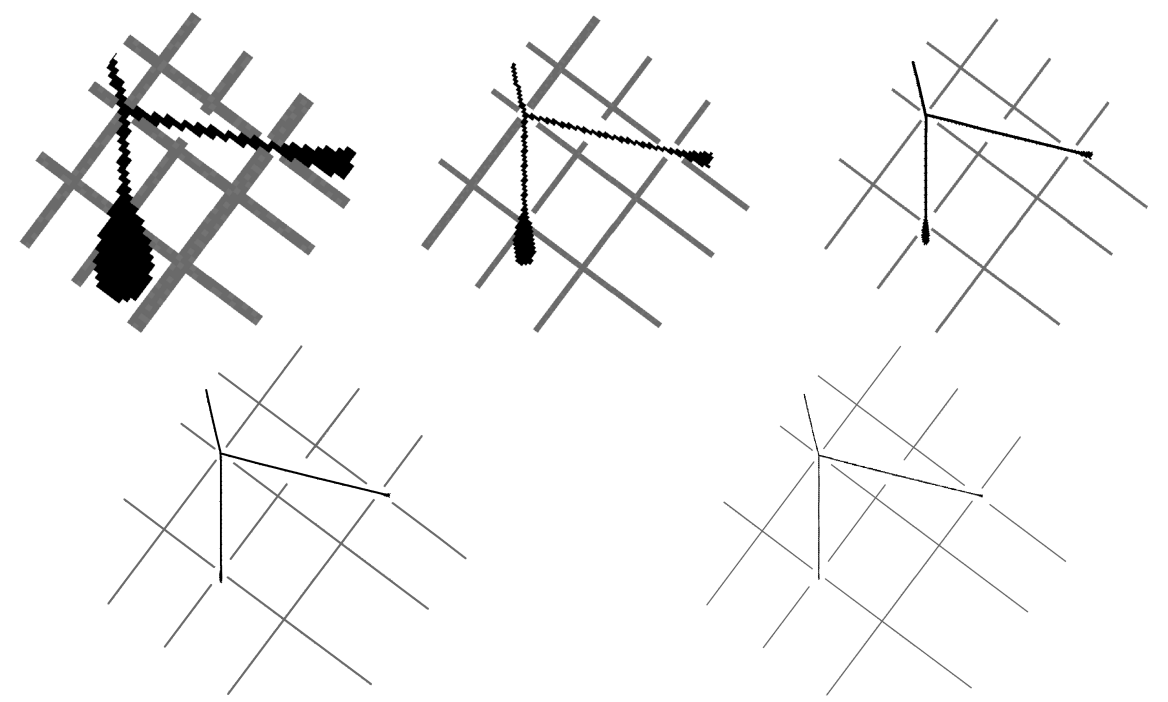

Figure 2. Approximations to the medial axis of the curve (1.11) using grid sizes $100 \times 100,200 \times 200,400 \times 400,800 \times 800$ and $1600 \times$ 1600. The Laplacian kernel $L^{(2)}$ has been used. The intersections of the dark diagonal grid lines indicate where the conjugate points and vertex must be. These lines are always a least two pixels wide with respect to the computational grid used.
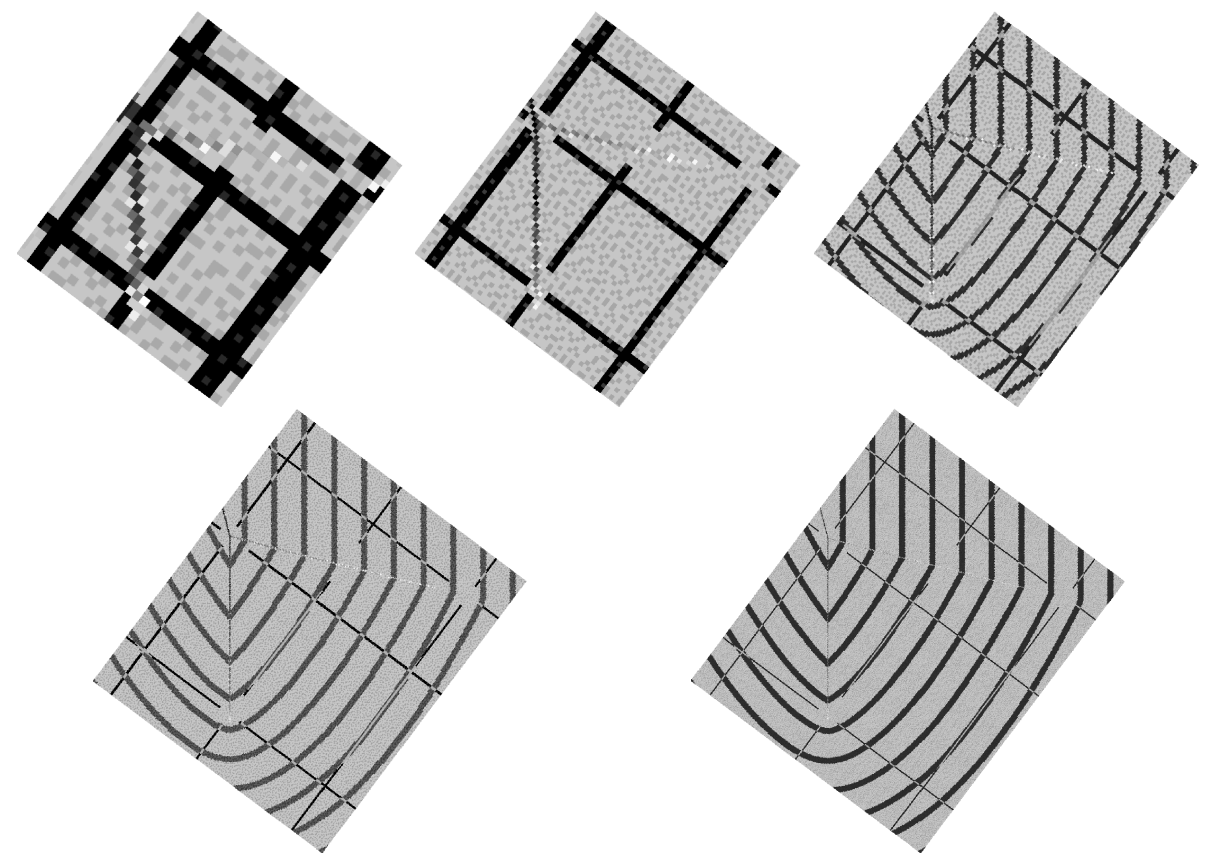

Figure 3. Approximations to the medial axis of the curve (1.11) using grid sizes $100 \times 100,200 \times 200,400 \times 400,800 \times 800$ and $1600 \times 1600$. The Laplacian kernel $L^{(3)}$ has been used. Contours of the distance function have been plotted for the finer grids. 
It has been conjectured that, in some generic sense, the conjugate locus consists only of folds and semi-cubical cusps [8]. We do know that in the two-dimensional plane the only two types of stable singularities are the fold singularities (of the Lagrangian map) and the cusps of the third canonical type of Lagrangian submanifold in two dimensions (when projected onto the surface, the critical points appear as semicubical cusps) [17, 5].

Consider the initial wavefront in $\mathbb{E}^{2}$

$$
\left\{\left(x_{1}, x_{1}^{2}-\frac{1}{2}\right) \mid x_{1} \leq 1\right\} \cup\left\{\left(1, x_{2}\right) \mid x_{2} \geq \frac{1}{2}\right\}
$$

and its inward normal motion at constant speed 1 . The distance map can be computed exactly, requiring only the real roots of a cubic polynomial in $x_{1}$ and $x_{2}$. The conjugate locus (first caustic) is given by

$$
\begin{aligned}
\left\{\left(x_{1},\left(\frac{27 x_{1}^{2}}{16}\right)^{1 / 3}\right) \mid x_{1} \geq-4\right\} & \\
\cup & \left\{\left(x_{1}, 1-\frac{x_{1}}{2}\right) \mid-4 \leq x_{1} \leq 1\right\} \\
\cup & \left\{\left(x_{1}, \frac{1}{2}\right) \mid x_{1} \leq 1\right\} .
\end{aligned}
$$

Note that the first set of this union does indeed include a semi-cubical cusp at $(0,0)$ which is turned towards the initial curve (and one can prove that it is indeed an endpoint of the cut locus).

The conjugate locus and distance map are illustrated in Figure 1. Note that it is difficult to judge where the cut locus might be from a mere visual inspection of the distance map.

The cut locus consists of a straight line segment from $(0,0)$ to $(0,3 / 4)$ and two other curves which are not straight, all three meeting at the vertex $(0,3 / 4)$. It has two endpoints (at $(0,0)$ and $(1,1 / 2))$. The cut points of our example can therefore be said to be generic except for the cusp at $(1,1 / 2)$.

Now we can apply our algorithm to this problem as a first check. The model problem's cut locus includes the straight line segment from $(0,0)$, the generic conjugate point, to $(0,3 / 4)$, the generic vertex. The direction of the $x_{2}$ axis is thereby given an artificial emphasis. If we check our algorithm using a grid aligned with the $x_{1}$ and $x_{2}$ axes, we can expect nongeneric behaviour at exactly the points which are most critical. Therefore, we used a grid rotated by $\arctan 3 / 4$.

The results for various grid sizes are shown in Figures 2 and 3 , for the Laplacian kernels $L^{(2)}$ and $L^{(3)}$, respectively. The nongeneric conjugate point creates most problems for both kernels. The generic conjugate point at $(0,0)$ is captured better by $L^{(3)} . L^{(2)}$ tends to "overshoot" past an actual conjugate point, making the cut locus appear longer than it actually is. The vertex is located well by both kernels. 

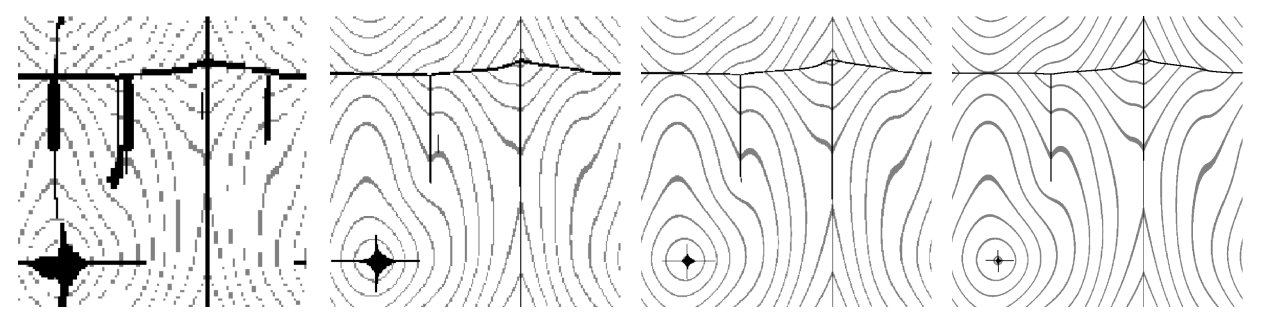

Figure 4. Convergence of the distance-map method using the Laplacian kernel $L^{(2)}$ to locate the cut locus. From left to right, the number of grid points in each direction is $100,200,400$, and 800 , respectively.

1.2. Tori: An example. We present an example of a Liouville surface diffeomorphic to the torus which is of elementary type (see Proposition 3.8 of [32]).

Let $\alpha_{1}=\alpha_{2}=2 \pi$ and the functions $f_{1}$ and $f_{2}$ be given by

$$
f_{1}\left(x_{1}\right)=\cos \left(4 x_{1}\right)+1.05 \text { and } f_{2}\left(x_{2}\right)=\cos \left(x_{2}\right)+1.05 \text {. }
$$

We will compute approximations to the cut locus from the point $(1,1)$ using regular square computational grids.

One can see how the cut locus approximations do appear to converge in Figure 4. What one cannot tell from this is whether the convergence is actually toward the cut locus at this level of graininess (i.e., we know that the algorithm should work in the limit of an infinitely fine grid, and the convergence shown in the case of the generic model is encouraging, but this does not mean that it will work for realistic grid sizes on a real problem). Fortunately, we are able to make a comparison with

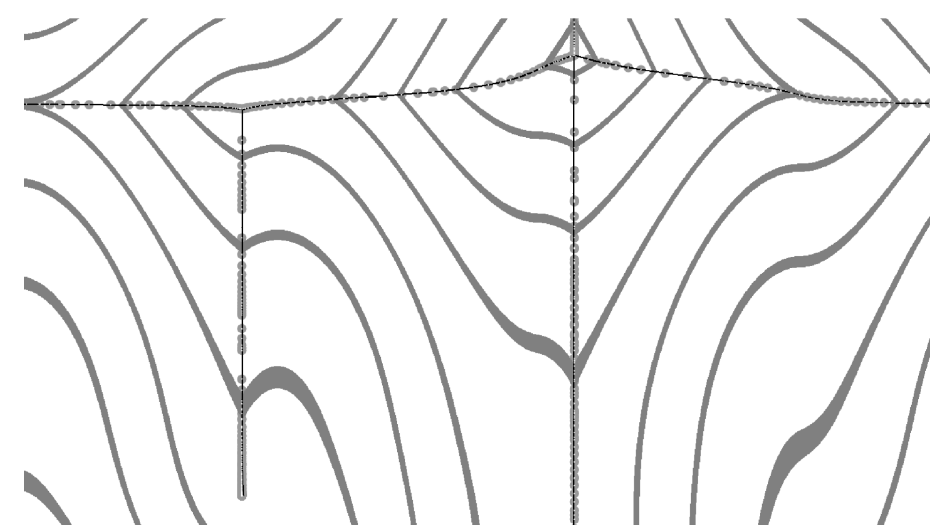

Figure 5. Part of a comparison of (i) an approximation of the cut locus from a point on a Liouville surface diffeomorphic to $T^{2}$ using the Laplacian kernel $L^{(2)}$ on a regular square grid of dimensions $1600 \times 1600$ and (ii) the software tool Loki with input error tolerance set to $10^{-5}$ for most of the points, but also $10^{-7}$ for the points defining the conjugate point near the lower left corner. The points computed by Loki are white, each with a much larger grey disk behind for highlighting purposes. 
the software tool Loki [59, which has shown itself to be reliable over a number of years. A close-up of the pleasing result of the comparison is shown in Figure 5 , Note that several components of the cut locus are straight line segments given by $x_{1}=$ const.

\section{Constructing Liouville surfaces on $S^{2}$}

2.1. Ellipsoids: An example. Here we will look at a single cut locus from a point on the ellipsoid

$$
\frac{y_{1}^{2}}{6}+y_{2}^{2}+\frac{y_{3}^{2}}{4}=1
$$

This example serves as a check of our software, since the general solution is known for ellipsoids [25, and also illustrates the nature of the coordinates $\left(x_{1}, x_{2}\right)$ on surfaces diffeomorphic to $S^{2}$.

In the notation of 32 we have $b_{1}=6, b_{2}=1, b_{3}=4$ and $\beta=\left(b_{3}-b_{2}\right) /\left(b_{1}-b_{2}\right)=$ $3 / 5$. The ellipsoid may be parametrized by $\mathbb{R} / 2 \pi \mathbb{Z} \times \mathbb{R} / \pi \mathbb{Z}=\left\{\theta_{1}, \theta_{2}\right\}$ as follows (equation (4.5) of [32]):

$$
\begin{aligned}
y_{1}\left(\theta_{1}, \theta_{2}\right), & =\sqrt{6} \cos \theta_{1} \sqrt{\frac{2}{5} \cos ^{2} \theta_{2}+\sin ^{2} \theta_{2}} \\
y_{2}\left(\theta_{1}, \theta_{2}\right) & =\cos \theta_{2} \sqrt{\frac{3}{5} \cos ^{2} \theta_{1}+\sin ^{2} \theta_{1}} \\
y_{3}\left(\theta_{1}, \theta_{2}\right) & =2 \sin \theta_{1} \sin \theta_{2} .
\end{aligned}
$$

See Figure 6. With respect to these coordinates, we have the metric

$g=\left(\frac{2}{5} \sin ^{2} \theta_{1}+\frac{3}{5} \sin ^{2} \theta_{2}\right)\left[\frac{4 \cos ^{2} \theta_{1}+6 \sin ^{2} \theta_{1}}{\frac{3}{5} \cos ^{2} \theta_{1}+\sin ^{2} \theta_{1}} d \theta_{1}^{2}+\frac{4 \cos ^{2} \theta_{2}+\sin ^{2} \theta_{2}}{\frac{2}{5} \cos ^{2} \theta_{2}+\sin ^{2} \theta_{2}} d \theta_{2}^{2}\right]$,

which is of the form (0.2).

We can define coordinates $x_{1}$ and $x_{2}$ by

$$
x_{1}(\theta)=\int_{0}^{\theta} \sqrt{\frac{4 \cos ^{2} t+6 \sin ^{2} t}{\frac{3}{5} \cos ^{2} t+\sin ^{2} t}} d t,
$$
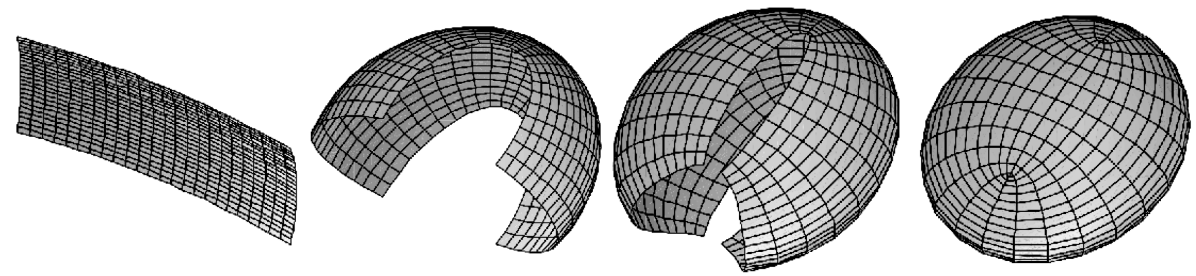

Figure 6 . The nature of the transformation from $[0,2 \pi) \times[0, \pi)$ to the surface of an ellipsoid. 
which can be expressed when $|\theta|<\pi / 2$ as

$$
\sqrt{6} \int_{0}^{\tan \theta} \frac{d x}{\sqrt{\left(\frac{3}{5}+x^{2}\right)\left(\frac{2}{3}+x^{2}\right)}}-\frac{\sqrt{6}}{3} \int_{0}^{\tan \theta} \frac{d x}{\sqrt{\left(\frac{3}{5}+x^{2}\right)\left(\frac{2}{3}+x^{2}\right)} \times\left(1+x^{2}\right)}
$$

(a sum of elliptic integrals of the first and the third kinds respectively; see Chapter 17 of [1] and also [49]), and

$$
x_{2}(\theta)=\int_{0}^{\theta} \sqrt{\frac{4 \cos ^{2} t+\sin ^{2} t}{\frac{2}{5} \cos ^{2} t+\sin ^{2} t}} d t
$$

and their inverses $\theta_{1}(x)$ and $\theta_{2}(x)$, respectively.

With respect to these coordinates, the metric is now

$$
g=\left(\frac{2}{5} \sin ^{2} \theta_{1}\left(x_{1}\right)+\frac{3}{5} \sin ^{2} \theta_{2}\left(x_{2}\right)\right)\left(d x_{1}^{2}+d x_{2}^{2}\right),
$$

which is clearly of the form (0.1).

Using the starting point $\left(\theta_{1}^{p}, \theta_{2}^{p}\right)$ defined by

$$
\left(y_{1}\left(\theta_{1}^{p}, \theta_{2}^{p}\right), y_{2}\left(\theta_{1}^{p}, \theta_{2}^{p}\right), y_{3}\left(\theta_{1}^{p}, \theta_{2}^{p}\right)\right)=\left(\frac{2 \sqrt{51}}{17}, \frac{2 \sqrt{51}}{17}, \frac{2 \sqrt{51}}{17}\right),
$$

which is the point

$$
\left(x_{1}^{p}, x_{2}^{p}\right) \approx(2.74126,1.44446),
$$

we can compute the cut locus using both Thaw [26] and our present algorithm. As was discovered in [26] and proven in [25], this cut locus is a subarc of a line of curvature $\left(\theta_{2}\right.$, and therefore also $x_{2}$ constant). Figure 7 shows that there is quantitative agreement. It is interesting to note that Thaw took two orders of magnitude longer to compute this approximation, although the errors made by the two computational tools seem to all intents and purposes equal, and despite the fact that we have not attempted to optimize the implementation of our present algorithm.

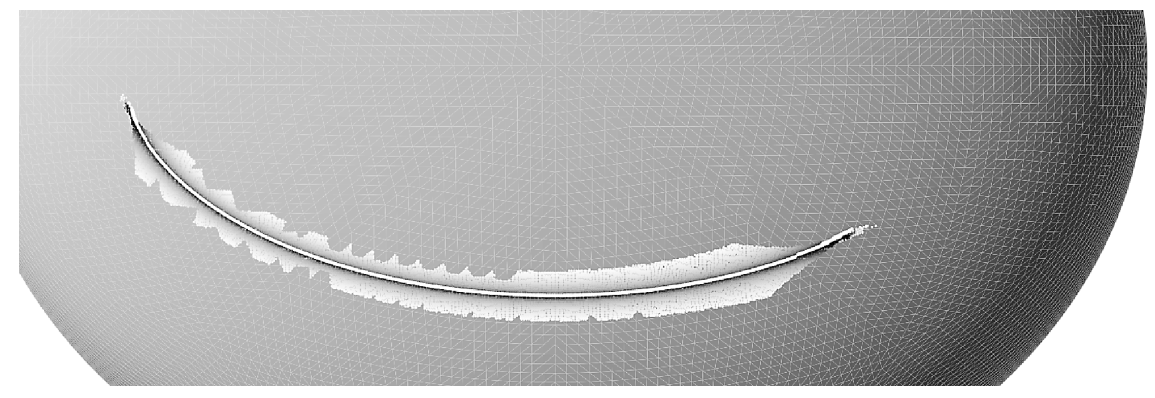

FiguRe 7. Comparison of approximations to the cut locus from a point on an ellipsoid computed by Thaw (dark central curve, fading to light grey) and the present algorithm (white curve). 


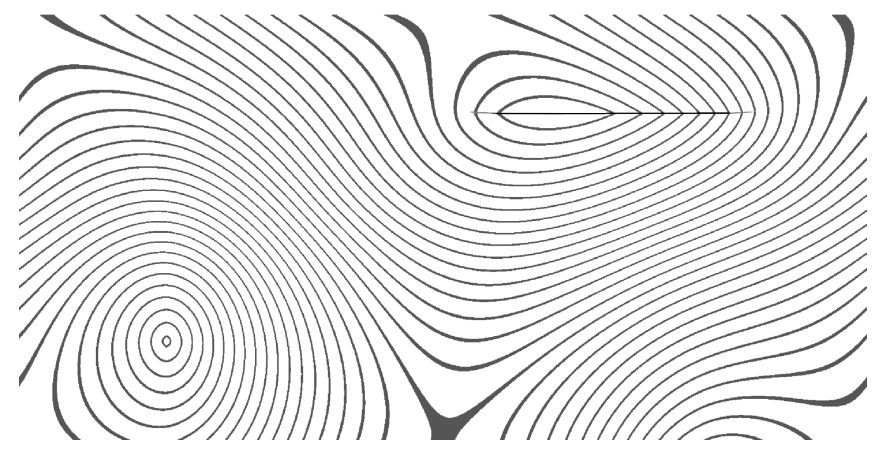

FiguRe 8. An approximation to the cut locus from a point on an ellipsoid computed by the present algorithm. The grey curves are geodesic circles centred around the starting point, located near the lower left corner. The horizontal black line diametrically opposite is the cut locus.

With respect to the coordinates $\left(x_{1}, x_{2}\right)$, the fundamental parallelogram is given by

$$
\left[0, x_{1}(2 \pi)\right) \times\left[0, x_{2}(\pi)\right) \approx[0,15.75669) \times[0,6.22718),
$$

and the cut locus from the point $\left(x_{1}^{p}, x_{2}^{p}\right)$ is a segment of the line

$$
\left\{(u, v) \mid v=x_{2}(\pi)-x_{2}^{p}\right\} .
$$

See Figure 8. Its rectangular shape is of course related to the surface of the ellipsoid as suggested by Figure 6 .

We wish to emphasize that the cut locus is a simple straight line segment in the natural coordinates $\left(x_{1}, x_{2}\right)$. It is precisely this type of behaviour which we have already seen for surfaces diffeomorphic to $T^{2}$, and will meet again on general Liouville surfaces diffeomorphic to $S^{2}$. Our conjectures derive from these simple observations.

2.2. More general compact analytic surfaces diffeomorphic to $S^{2}$. In the following we will frequently refer to the paper "Compact Liouville Surfaces" [32].

Our aim is to describe practical numerical methods for constructing quadruples of two positive real constants and two real analytic functions $\left(\alpha_{1}, \alpha_{2}, f_{1}, f_{2}\right)$ which define real compact analytic Liouville surfaces whose underlying manifold is $S^{2}$. Note that the same quadruple defines the double covering of a Louville surface whose underlying manifold is the real projective plane $\mathbb{R P}^{2}$ (Corollary 3.7 of [32]). The conditions which must be met are conditions (3.1) and (3.2) of [32. We will assume that the pair $\left(\alpha_{1}, \alpha_{2}\right)$ is given, and provide a basis for the set of suitable functions $f_{1}$ and $f_{2} . f_{2}$ is completely determined by $f_{1}$ in the analytic case (Corollary 3.6 of [32]).

$f_{1}$ is identified with a $C^{\infty}$ even periodic function on $\mathbb{R}$ with period $\alpha_{1} / 2$ such that $f_{1}^{\prime \prime}(0)>0, f_{1}(0)=0$, and $f_{1}(t)>0$ if $t \neq 0\left(\bmod \left(\alpha_{1} / 2\right) \mathbb{Z}\right)$. Corresponding conditions apply to $f_{2}$, which has the period $\alpha_{2} / 2$. 
The formal Taylor expansions around $t=0$ (denoted by $\sim$ ) of $f_{1}$ and $f_{2}$ must be related via

$$
f_{1}(t) \sim \sum_{k \geq 1} a_{k} t^{2 k} \quad \Leftrightarrow \quad f_{2}(t) \sim \sum_{k \geq 1}(-1)^{k-1} a_{k} t^{2 k} .
$$

This condition can be satisfied using a suitable holomorphic function $j: \mathbb{C} \rightarrow \mathbb{R}$ if we identify

$$
f_{1}(t)=j(t) \quad \text { and } \quad f_{2}(t)=-j(\sqrt{-1} t)
$$

since, formally,

$$
\sum_{k \geq 1} a_{k}(\sqrt{-1} t)^{2 k}=-\sum_{k \geq 1} a_{k}(-1)^{k-1} t^{2 k}
$$

See Corollary 3.6 of 32 .

We are therefore looking for doubly periodic (elliptic) functions. Since the first Liouville theorem (Theorem 1 of Section 9 of Part II of [34) tells us that there is no nonconstant doubly periodic entire function, it is not immediately clear which functions to use as the basis of our construction. Although it is certainly the case that any doubly periodic function can be expressed in terms of rational functions of the Weierstrass function and its derivatives (Theorem stated in Section 2.3 of [49]), we have found it more convenient to take the route of Jacobian elliptic functions.

The function

$$
j_{n}(t)=\frac{1-\operatorname{sn}(n t+K(m) \mid m)}{2}
$$

(using the notation of [1, where sn is a Jacobian elliptic function, $K$ is the complete elliptic integral of the first kind, $0<m<1$ is the parameter, and $n$ is a positive integer) is doubly periodic, and both $j_{n}(t)$ and $-j_{n}(\sqrt{-1} t)$ are real-valued and bounded (for fixed $m$ ). Indeed,

$$
f_{1}^{(n)}(t)=\frac{1-\operatorname{sn}(n t+K(m) \mid m)}{2}=\frac{1-\frac{\operatorname{cn}(n t \mid m)}{\operatorname{dn}(n t \mid m)}}{2}=\frac{1-\operatorname{cd}(n t \mid m)}{2}
$$

with

$$
\alpha_{1}=8 K(m)
$$

and

$$
f_{2}^{(n)}(t)=-\frac{1-\operatorname{sn}(\sqrt{-1} n t+K(m) \mid m)}{2}=\frac{\operatorname{nd}(n t \mid 1-m)-1}{2}
$$

with

$$
\alpha_{2}=4 K(1-m)
$$

satisfy the conditions (3.1) and (3.2) of 32 for all $0<m<1$, as can easily be verified. Note that the $f_{1}^{(n)}$ are normalized to have the range $[0,1]$. Since these conditions are linear, and since the functions $f_{1}^{(n)}$ are a basis for the set of analytic functions $f_{1}$ satisfying these conditions, we may write any suitable analytic functions as

$$
F_{1}(t)=\sum_{n=1}^{\infty} \ell_{n} f_{1}^{(n)}(t) \quad \text { and } \quad F_{2}(t)=\sum_{n=1}^{\infty} \ell_{n} f_{2}^{(n)}(t)
$$




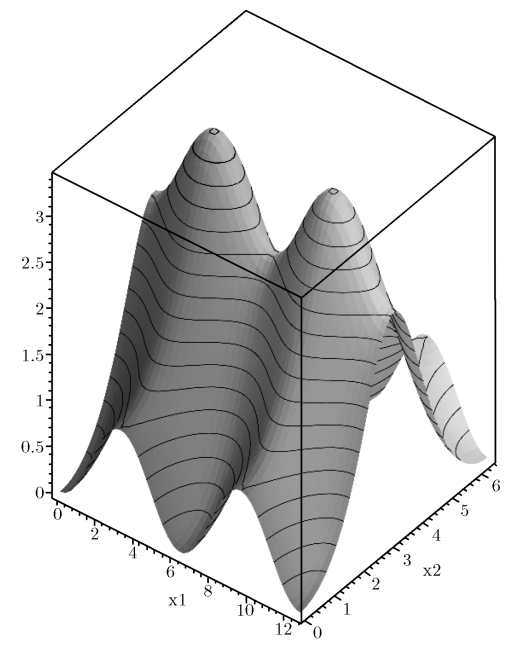

Figure 9. $f_{1}^{(1)}\left(x_{1}\right)+f_{2}^{(1)}\left(x_{2}\right)$ plotted for $\alpha_{1}=\alpha_{2} \approx 12.6604$.

where the real coefficients $\ell_{n}$ must be chosen such that $F_{1}^{\prime \prime}(0)>0, F_{2}^{\prime \prime}(0)>0$, $F_{1}(t)>0$ if $t \neq 0\left(\bmod \left(\alpha_{1} / 2\right) \mathbb{Z}\right)$, and also $F_{2}(t)>0$ if $t \neq 0\left(\bmod \left(\alpha_{2} / 2\right) \mathbb{Z}\right)$. A necessary condition is that

$$
\ell_{1}>0 \wedge 0<\sum_{n=1}^{\infty} n^{2} \ell_{n}<\infty
$$

Given a pair of positive constants $\alpha_{1}$ and $\alpha_{2}$, we can construct valid quadruples $\left(\alpha_{1}, \alpha_{2}, f_{1}, f_{2}\right)$ by choosing the unique value of the parameter $m_{\alpha}$ which satisfies

$$
\frac{2 K\left(m_{\alpha}\right)}{K\left(1-m_{\alpha}\right)}=\frac{\alpha_{1}}{\alpha_{2}}
$$

and defining the positive constant $c_{\alpha}$ by

$$
c_{\alpha}=\frac{\alpha_{1}}{8 K\left(m_{\alpha}\right)} .
$$

Then we can make use of the equivalence of quadruples $\left(\alpha_{1}, \alpha_{2}, f_{1}, f_{2}\right)$ and $\left(c \alpha_{1}, c \alpha_{2}\right.$, $\left.f_{1} / c^{2}, f_{2} / c^{2}\right)$ with $c>0$ to write

$$
\left(8 c_{\alpha} K\left(m_{\alpha}\right), 4 c_{\alpha} K\left(1-m_{\alpha}\right),\left(1 / c_{\alpha}^{2}\right) F_{1},\left(1 / c_{\alpha}^{2}\right) F_{1}\right) \equiv\left(\alpha_{1}, \alpha_{2}, f_{1}, f_{2}\right)
$$

and

$$
\left(\alpha_{1}, \alpha_{2}, f_{1}, f_{2}\right) \text { is equivalent to }\left(8 K\left(m_{\alpha}\right), 4 K\left(1-m_{\alpha}\right), F_{1}, F_{2}\right) \text {. }
$$

2.3. Computing $K\left(m_{\alpha}\right), K\left(1-m_{\alpha}\right)$, and $m_{\alpha}$ from the ratio $\alpha_{1} / \alpha_{2}$. The following computations will only need to be done once for any given ratio of $\alpha_{1} / \alpha_{2}$.

From equation (2.22), we can write the nome

$$
q(m)=\exp \left(\frac{-\pi K(1-m)}{K(m)}\right)
$$


in terms of $\alpha_{1} / \alpha_{2}$ :

$$
q\left(m_{\alpha}\right)=\exp \left(-2 \pi \frac{\alpha_{2}}{\alpha_{1}}\right)
$$

We can then compute $K\left(m_{\alpha}\right)$ and $K\left(1-m_{\alpha}\right)$ using this and equation 17.3 .22 of [1:

$$
K\left(m_{\alpha}\right)=2 \pi\left[\frac{1}{4}+\sum_{k=1}^{\infty} \frac{q^{k}\left(m_{\alpha}\right)}{1+q^{2 k}\left(m_{\alpha}\right)}\right]=\pi\left[\frac{1}{2}+\sum_{k=1}^{\infty} \frac{1}{\cosh \left(2 k \pi \frac{\alpha_{2}}{\alpha_{1}}\right)}\right]
$$

and

$$
K\left(1-m_{\alpha}\right)=\frac{2 K\left(m_{\alpha}\right) \alpha_{2}}{\alpha_{1}}
$$

To compute $m_{\alpha}$ from $K\left(m_{\alpha}\right)$, we can use bisection to solve for $m$ in $K(m)=$ $K\left(m_{\alpha}\right)$, where $K(m)$ is calculated using the arithmetic-geometric mean (see Section 17.6 of [1]). In our case, we have

$$
a_{0}=1, \quad b_{0}=\sqrt{1-m}, \quad a_{i+1}=\frac{a_{i}+b_{i}}{2}, \quad b_{i+1}=\sqrt{a_{i} b_{i}}
$$

for all nonnegative integers $i$. This process will quickly converge. When $a_{N-1}=a_{N}$ and $b_{N-1}=b_{N}$ (to numerical precision), then

$$
K(m)=\frac{\pi}{2 a_{N}} .
$$

2.4. Trigonometric series expansions. While there are many well-known algorithms for the rapid computation of elliptic functions (see [1] and Section 5.3 of 39 for an overview), we have decided to instead make use of trigonometric series expansions to avoid catastrophic cancellation in the differences $1-\operatorname{cd}(n t \mid m)$ and $\operatorname{nd}(n t \mid 1-m)-1$. The series expansions will provide high accuracy even close to zeros of these functions.

In the following, we will use the abbreviations $K \equiv K(m), K^{\prime} \equiv K(1-m)$, and $q \equiv q(m)$, where $q$ is the nome. Note that $0<m<1$ implies $0<q<1$.

We have the series expansion

$$
\operatorname{cd}(u \mid m)=\frac{2 \pi}{\sqrt{m} K} \sum_{n=0}^{\infty} \frac{(-1)^{n} q^{n+1 / 2}}{1-q^{2 n+1}} \cos \left\{\left(n+\frac{1}{2}\right) \frac{\pi u}{K}\right\}
$$

from [1. Using the fact that $\operatorname{cd}(0 \mid m)=1$, we have

$$
\operatorname{cd}(0 \mid m)=\frac{2 \pi}{\sqrt{m} K} \sum_{n=0}^{\infty} \frac{(-1)^{n} q^{n+1 / 2}}{1-q^{2 n+1}}=1 \Rightarrow \sum_{n=0}^{\infty} \frac{(-1)^{n} q^{n+1 / 2}}{1-q^{2 n+1}}=\frac{\sqrt{m} K}{2 \pi} .
$$


Now

$$
\begin{aligned}
\frac{1}{(2.33)} & =\frac{\pi}{\sqrt{m} K} \sum_{n=0}^{\infty} \frac{(-1)^{n+1} q^{n+1 / 2}}{1-q^{2 n+1}} \cos \left\{\left(n+\frac{1}{2}\right) \frac{\pi u}{K}\right\}+\frac{1}{2} \\
& =\frac{\pi}{\sqrt{m} K}\left[\sum_{n=0}^{\infty} \frac{(-1)^{n+1} q^{n+1 / 2}}{1-q^{2 n+1}}\left(\cos \left\{\left(n+\frac{1}{2}\right) \frac{\pi u}{K}\right\}-1\right)-\frac{\sqrt{m} K}{2 \pi}\right]+\frac{1}{2} \\
& =\frac{2 \pi}{\sqrt{m} K} \sum_{n=0}^{\infty} \frac{(-1)^{n} q^{n+1 / 2}}{1-q^{2 n+1}} \sin ^{2}\left\{\left(n+\frac{1}{2}\right) \frac{\pi u}{2 K}\right\} \\
(2.34) & =\frac{\pi}{\sqrt{m} K} \sum_{n=0}^{\infty} \frac{(-1)^{n}}{\sinh \left(\frac{(n+1 / 2) \pi K^{\prime}}{K} \sin ^{2}\left\{\left(n+\frac{1}{2}\right) \frac{\pi u}{2 K}\right\} .\right.}
\end{aligned}
$$

Let us now turn to $f_{2}$. We also have the series expansion

$$
\operatorname{nd}(u \mid m)=\frac{\pi}{2 \sqrt{1-m} K}+\frac{2 \pi}{\sqrt{1-m} K} \sum_{n=1}^{\infty} \frac{(-1)^{n} q^{n}}{1+q^{2 n}} \cos \left\{\frac{n \pi u}{K}\right\}
$$

from [1. Since $\operatorname{nd}(0 \mid m)=1$, we have

$$
\operatorname{nd}(0 \mid m)=\frac{\pi}{2 \sqrt{1-m} K}+\frac{2 \pi}{\sqrt{1-m} K} \sum_{n=1}^{\infty} \frac{(-1)^{n} q^{n}}{1+q^{2 n}}=1,
$$

from which it follows that

$$
\sum_{n=1}^{\infty} \frac{(-1)^{n} q^{n}}{1+q^{2 n}}=\frac{\sqrt{1-m} K}{2 \pi}-\frac{1}{4}
$$

and therefore

$$
\begin{aligned}
\frac{\operatorname{nd}(u \mid m)-1}{2} & =\frac{\pi}{\sqrt{1-m} K}\left[\frac{1}{4}+\sum_{n=1}^{\infty} \frac{(-1)^{n} q^{n}}{1+q^{2 n}} \cos \frac{n \pi u}{K}\right]-\frac{1}{2} \\
& =\frac{\pi}{\sqrt{1-m} K} \sum_{n=1}^{\infty} \frac{(-1)^{n+1}}{\cosh \left(\frac{n \pi K^{\prime}}{K}\right)} \sin ^{2} \frac{n \pi u}{2 K}
\end{aligned}
$$

2.5. Numerical evaluation of $f_{1}^{(n)}$ and $f_{2}^{(n)}$ and their derivatives. We have

$$
f_{1}^{(n)}(t)=\frac{\pi}{\sqrt{m} K(m)} \sum_{k=0}^{\infty} \frac{(-1)^{k}}{\sinh \left(\frac{(k+1 / 2) \pi K(1-m)}{K(m)}\right)} \sin ^{2}\left\{\left(k+\frac{1}{2}\right) \frac{n \pi t}{2 K(m)}\right\}
$$

and

$$
\begin{aligned}
f_{2}^{(n)}(t) & =\frac{\operatorname{nd}(t \mid 1-m)-1}{2} \\
& =\frac{\pi}{\sqrt{m} K(1-m)} \sum_{k=1}^{\infty} \frac{(-1)^{k+1}}{\cosh \left(\frac{k \pi K(m)}{K(1-m)}\right)} \sin ^{2} \frac{n k \pi t}{2 K(1-m)} .
\end{aligned}
$$


Expansions (2.34) and (2.39) do not depend upon any cancellation of terms to become zero, and are therefore preferable in comparison with (2.33) and (2.38). We use the expansions (2.40) and (2.41) to compute $f_{1}^{(n)}$ and $f_{2}^{(n)}$, respectively, when the nome $q(m)$ and its complement $q(1-m)$ are both small.

For first and second derivatives of $f_{1}^{(n)}$ and $f_{2}^{(n)}$, the expansions (2.33) and (2.38) are easier to deal with. We find that

$$
\frac{d f_{1}^{(n)}(t)}{d t}=\frac{n \pi^{2}}{2 \sqrt{m} K^{2}(m)} \sum_{k=0}^{\infty} \frac{(-1)^{k}\left(k+\frac{1}{2}\right)}{\sinh \frac{\left(k+\frac{1}{2}\right) \pi K(1-m)}{K(m)}} \sin \left\{\left(k+\frac{1}{2}\right) \frac{n \pi t}{K(m)}\right\}
$$

$$
\begin{aligned}
& \frac{d^{2} f_{1}^{(n)}(t)}{d t^{2}}=\frac{n^{2} \pi^{3}}{2 \sqrt{m} K^{3}(m)} \sum_{k=0}^{\infty} \frac{(-1)^{k}\left(k+\frac{1}{2}\right)^{2}}{\sinh \frac{\left(k+\frac{1}{2}\right) \pi K(1-m)}{K(m)}} \cos \left\{\left(k+\frac{1}{2}\right) \frac{n \pi t}{K(m)}\right\}, \\
& \frac{d f_{2}^{(n)}(t)}{d t}=\frac{n \pi^{2}}{2 \sqrt{m} K^{2}(1-m)} \sum_{k=1}^{\infty} \frac{(-1)^{k+1} k}{\cosh \frac{k \pi K(m)}{K(1-m)}} \sin \frac{k n \pi t}{K(1-m)}
\end{aligned}
$$

and

$$
\frac{d^{2} f_{2}^{(n)}(t)}{d t^{2}}=\frac{n^{2} \pi^{3}}{2 \sqrt{m} K^{3}(1-m)} \sum_{k=1}^{\infty} \frac{(-1)^{k+1} k^{2}}{\cosh \frac{k \pi K(m)}{K(1-m)}} \cos \frac{k n \pi t}{K(1-m)} .
$$

If we wish to have 18 significant figures (considering truncation error only), it suffices to require that $0.02<m<0.98$ and only add up to $k=35$ in all of the above expansions, since this ensures that both $q(m)$ and $q(1-m)$ are less than 0.23 . This range allows us to deal with ratios $\alpha_{1} / \alpha_{2} \in[0.9414,4.2491]$ (see equation (2.22) $)$.

For $m$ outside this range, the descending Landen transformation (also known as Gauss' transformation) or the ascending Landen transformation 1] can be repeated until $m$, which we assume to always be in the open interval $(0,1)$, is within $[0.02,0.98]$.

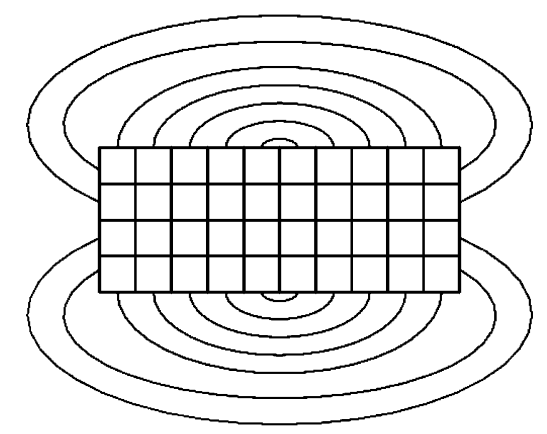

FIgURE 10. A sample $8 \times 4$ computational grid showing border connectivity. The grid covers the domain $\left[0, \alpha_{1}\right) \times\left[0, \alpha_{2} / 2\right)$. 
2.6. Computational grid. We use a regular rectangular grid of $N_{1}$ and $N_{2}$ points in the directions of the $x_{1}$ and $x_{2}$ axes, respectively. The grid covers the domain

$$
\left[0, \alpha_{1}\right) \times\left[0, \frac{\alpha_{2}}{2}\right)=\left[0,8 K\left(m_{\alpha}\right)\right) \times\left[0,2 K\left(1-m_{\alpha}\right)\right) .
$$

To ensure that the grid cells are almost square, we set

$$
N_{1}=\left\lfloor 2 N_{2} \frac{\alpha_{1}}{\alpha_{2}}+\frac{1}{2}\right\rfloor \text {. }
$$

We have

$$
\Delta x_{1}=\frac{8 K\left(m_{\alpha}\right)}{N_{1}} \quad \text { and } \quad \Delta x_{2}=\frac{2 K\left(1-m_{\alpha}\right)}{N_{2}} .
$$

For $i_{1} \in\left\{0,1, \ldots, N_{1}-1\right\}$ and $i_{2} \in\left\{0,1, \ldots, N_{2}-1\right\}$, we define the mapping to canonical coordinates by

$$
x_{1}=\frac{8 K\left(m_{\alpha}\right)\left(i_{1}+\frac{1}{2}\right)}{N_{1}}
$$

and

$$
x_{2}=\frac{2 K\left(1-m_{\alpha}\right)\left(i_{2}+\frac{1}{2}\right)}{N_{2}} .
$$

Note that care has been taken not to map any grid point to any of the four points at which the metric is singular $\left(F_{1}\left(x_{1}\right)+F_{2}\left(x_{2}\right)=0\right)$.

In accordance with Section 3 of 32] (and Figure 6) the edges are identified as illustrated in Figure 10.

2.7. First "generic" experiments. Now we have a set of computational tools which allow us to approximate the cut loci from points on compact Liouville surfaces diffeomorphic to $S^{2}$.

As a first experiment, let us take the "generic" surface given by

$$
F_{1}(x)=0.1 f_{1}^{(1)}(x)+0.25 f_{1}^{(2)}(x)+0.111 f_{1}^{(3)}(x)-0.0625 f_{1}^{(4)}(x)+0.01 f_{1}^{(5)}(x)
$$

(from which $F_{2}$ is uniquely determined, having the same numerical coefficients in its expansion), and

$$
\frac{\alpha_{1}}{\alpha_{2}}=1.6
$$

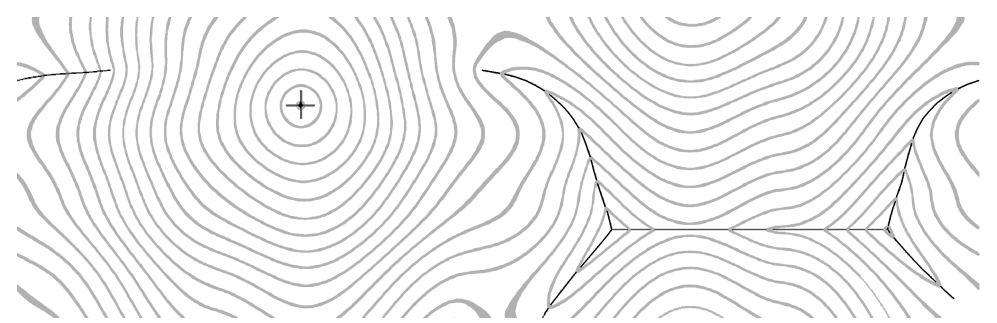

Figure 11. The cut locus from the point $(4,3)$ on the surface given by $F_{1}(x)=0.1 f_{1}^{(1)}(x)+0.25 f_{1}^{(2)}(x)+0.111 f_{1}^{(3)}(x)-$ $0.0625 f_{1}^{(4)}(x)+0.01 f_{1}^{(5)}(x)$ with $\alpha_{1} / \alpha_{2}=1.6$, computed on a grid of dimensions $2560 \times 800$. 
from which we compute $m_{\alpha} \approx 0.27051, K\left(m_{\alpha}\right) \approx 1.69703, K\left(1-m_{\alpha}\right) \approx 2.12129$, $\alpha_{1} \approx 13.57627$ and $\alpha_{2} \approx 8.48517$. This surface has points of both positive and negative Gaussian curvature. For the starting point $(4,3)$, the Laplacian kernel $L^{(2)}$ (equation (1.9) - the starting point will be visible), and using 2560 grid points in the $x_{1}$ direction and 800 grid points in the $x_{2}$ direction, we obtain Figure 11 . Note that one part of the cut locus consists of a line segment with $x_{2}=$ const, but there is no further structure which would motivate a conjecture.

A second experiment with another "generic" surface, which also has points of both positive and negative Gaussian curvature (Figure 12), shows some shortcomings of our method to approximate the cut locus using the Laplacian kernel $L^{(2)}$ alone. The cut locus appears to consist of a number of disjoint arcs. This is not what one would like. Of course we know that the cut locus must be a connected 1-dimensional complex [43]. The experimental difficulty arises from the fact that the two geodesics meeting at an ordinary cut point may be almost tangential to each other (they can never actually be tangential where they intersect, since then they would have to be the same geodesic). In this second example, the starting point and curvature conspire to induce a sequence of subarcs of the cut locus whose points are each the result of two geodesics meeting at some very small angle.

It must however be the case that we can capture the cut locus by using a fine enough grid. The grid used in Figure 12 was $1600 \times 800$. In Figure 13 we can see the result, computed on a grid of dimensions $6400 \times 3200$, where we have used the Laplacian kernel

$$
\left|L\left(x_{1}, x_{2}, d^{2}\left(\left(x_{1}^{p}, x_{2}^{p}\right),(\cdot, \cdot)\right)\right)\right|^{0.01}
$$

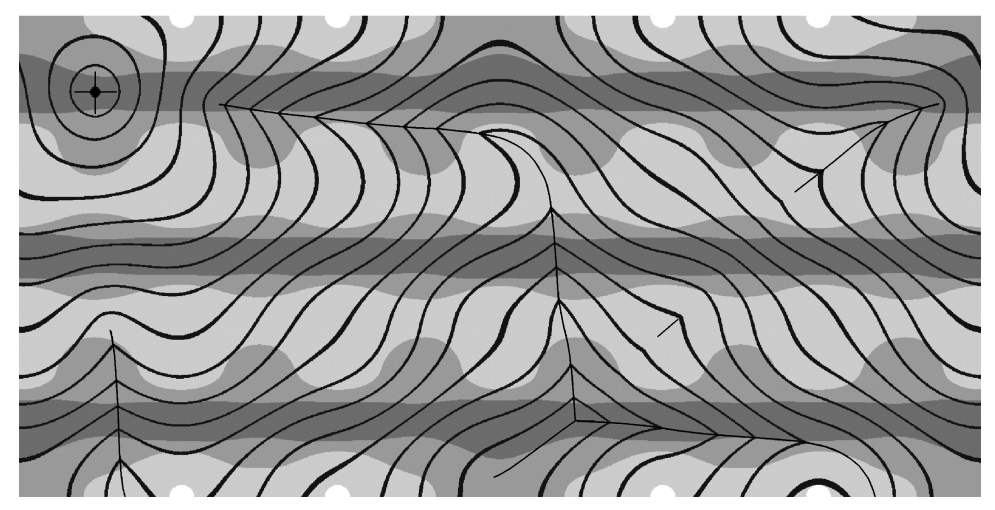

Figure 12. A plot of the cut locus (black) from a point (large black spot with crosshairs) with equidistant curves (very dark grey) and Gaussian curvature (white for very negative, light grey for negative, grey for positive, and dark grey for very positive) on the surface given by $F_{1}\left(x_{1}\right)=0.1 f_{1}^{(1)}\left(x_{1}\right)+0.2 f_{1}^{(2)}\left(x_{1}\right)+f_{1}^{(3)}\left(x_{1}\right)$ with $\alpha_{1}=\alpha_{2} \approx 12.6604$ and the starting point at $(1,5.3302)$. Note that the cut locus appears to consist of a number of disjoint arcs. This is an artifact of the approximation method used, which attempts to deduce the position of the cut locus using only local operations on the distance map. 


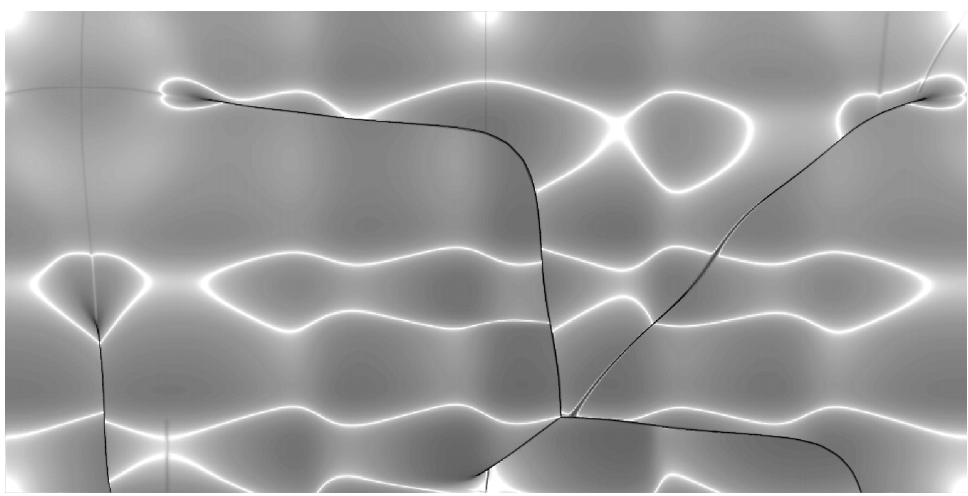

Figure 13. Recomputation of the cut locus approximation of Figure 12 using a finer computational grid $(6400 \times 3200$ instead of $1600 \times 800)$ and a less selective Laplacian kernel $\left|L\left(x_{1}, x_{2}, d^{2}\left(\left(x_{1}^{p}, x_{2}^{p}\right),(\cdot, \cdot)\right)\right)\right|^{0.01}$. The contrast of the computed image has also been enhanced in a post-processing step. The entire cut locus is now visible.

with no threshold on which points are shaded (all points are shaded according to their normalized values). One can now see the cut locus as a connected tree.

From an experimental point of view, this is still an unpleasant situation to be in, since one is left with the choice of either having to use large grids, or having to deal with possible confusion concerning which points are endpoints of the cut locus and so on. It is for this reason that we have included a routine which computes an approximation to the conjugate locus. We can then see which points are endpoints, since they must each coincide with a cusp of the conjugate locus.

2.8. Computing an approximation to the conjugate locus. Here we will use a Lagrangian method, since it offers high accuracy at precisely the cusps of the conjugate locus (where the geodesics are converging) which will give us the positions of the endpoints of the cut locus. Robust Eulerian methods do also exist [4, which are more efficient but less suited to our particular needs.

Our experience from earlier work 60. was, perhaps surprisingly, that it can be cumbersome to make use of the equations of geodesics in their integrated forms in numerical software. This is due to the necessity of having to determine when one should change direction or coordinates, or both. Instead, we make use of the standard geodesic equations (equation (2.2) of Section 2 of Chapter II of [54]) which take the form

$$
\begin{aligned}
& \ddot{y}_{1}=\frac{\frac{1}{2} \dot{f}_{1}\left(y_{1}\right) \dot{y}_{1}^{2}+\dot{f}_{2}\left(y_{2}\right) \dot{y}_{1} \dot{y}_{2}-\frac{1}{2} \dot{f}_{1}\left(y_{1}\right) \dot{y}_{2}^{2}}{f_{1}\left(y_{1}\right)+f_{2}\left(y_{2}\right)} \\
& \ddot{y}_{2}=\frac{\frac{1}{2} \dot{f}_{2}\left(y_{2}\right) \dot{y}_{2}^{2}+\dot{f}_{1}\left(y_{1}\right) \dot{y}_{1} \dot{y}_{2}-\frac{1}{2} \dot{f}_{2}\left(y_{2}\right) \dot{y}_{1}^{2}}{f_{1}\left(y_{1}\right)+f_{2}\left(y_{2}\right)}
\end{aligned}
$$

in the case of a Liouville surface, where dots denote differentiation (with respect to arclength in the case of $y_{1}$ and $y_{2}$ ). 
These can be integrated using the classical fourth-order Runge-Kutta method (Algorithm (9.76) in [55]). We found it useful to have an adaptive step-size.

It is a well-known fact that one way to find conjugate points is to look for zeros of Jacobi fields, which are solutions of the Jacobi equation (see equations (2.19) and (2.20) in Chapter II of [54]). Since we are dealing only with two-dimensional surfaces, we have actually used this equation's simpler predecessor, the Gauss equation (contained in Section 19 of his "General Remarks on Curved Surfaces" 18, published in 1827; it appears as equation (3.9) in 7] and equation (4) in [51, which also includes a detailed description of its derivation and meaning, as well as historical detail concerning its generalization to the modern form referred to above):

$$
\frac{d^{2} Y(s)}{d s^{2}}+G(\gamma(s, \theta)) Y(s)=0
$$

where $\gamma$ is a geodesic parametrized by arclength $s$ and initial angle $\theta, G$ the Gaussian curvature, and the function $Y: \mathbb{R} \rightarrow \mathbb{R}$ is the geodesic deviation which satisfies

$$
Y(0)=0 \quad \text { and }\left.\quad \frac{d Y(s)}{d s}\right|_{s=0}=1 .
$$

One can then integrate these equations along a geodesic, returning the location of the first zero to the position of the (first) conjugate point along that geodesic.

On a Liouville surface, the Gaussian curvature is

$$
G\left(x_{1}, x_{2}\right)=\frac{\left(\frac{d f_{1}\left(x_{1}\right)}{d x_{1}}\right)^{2}+\left(\frac{d f_{2}\left(x_{2}\right)}{d x_{2}}\right)^{2}}{2\left(f_{1}\left(x_{1}\right)+f_{2}\left(x_{2}\right)\right)^{3}}-\frac{\frac{d^{2} f_{1}\left(x_{1}\right)}{d x_{1}^{2}}+\frac{d^{2} f_{2}\left(x_{2}\right)}{d x_{2}^{2}}}{2\left(f_{1}\left(x_{1}\right)+f_{2}\left(x_{2}\right)\right)^{2}} .
$$

Figure 14 shows a computation performed with the same grid size as used for Figure 12 but with the conjugate locus marked. The four endpoints of the cut locus are now clearly identified.

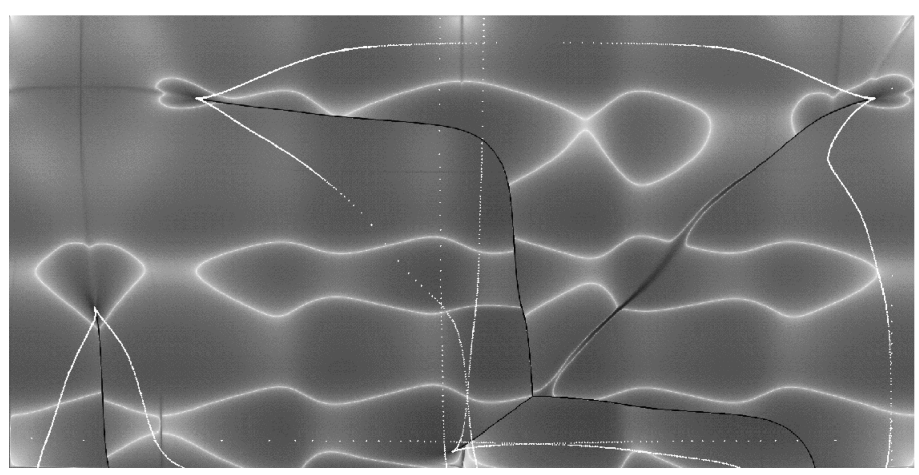

FIgURE 14. Recomputation of the cut locus approximation of Figure 12 using the same computational grid $(1600 \times 800)$ and the less selective Laplacian kernel used for Figure 13. Here conjugate points (white) have been added. This clearly helps in locating the four endpoints of the cut locus. 


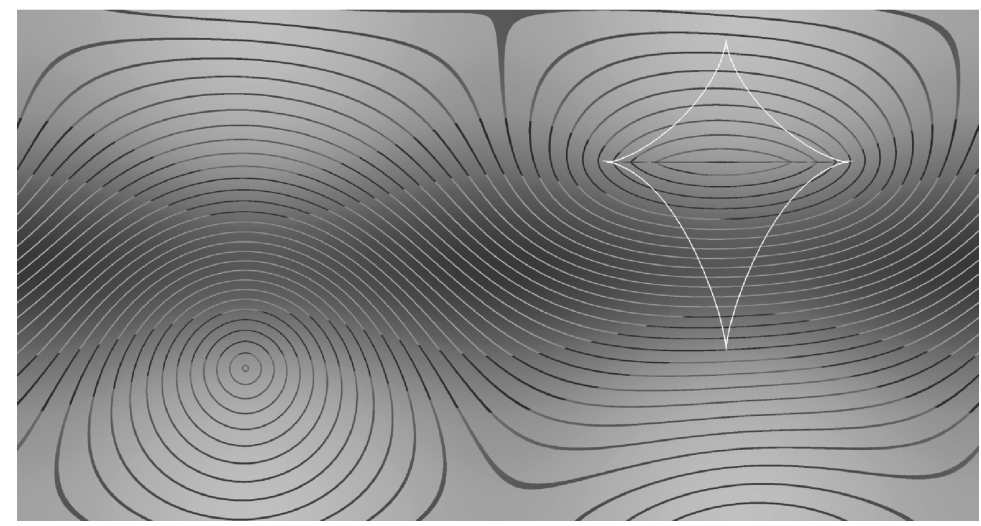

Figure 15. The surface given by $F_{1}(x)=f_{1}^{(1)}(x)+$ $0.00002 f_{1}^{(3)}(x), m_{\alpha}=0.02, \alpha_{1} \approx 12.62992$, and $\alpha_{2} \approx 13.41656$ with cut locus (horizontal dark line segment) and conjugate locus (in white) from the point $(3,2)$. The computational grid used has the dimensions $3012 \times 1600$. This example is typical, and clearly shows the straight cut locus and four cusps of the conjugate locus.

\section{EXAMPLES LEADING TO THE MAIN CONJECTURE}

Here we will present the results of a few of the many experiments we have performed, concentrating first on surfaces of the form $F_{1}(x)=f_{1}^{(1)}(x)+$ "small, higherorder perturbations".

A very representative example, illustrated in Figure [15, is of the conjugate and cut loci from the point $(3,2)$ on the surface given by $F_{1}(x)=f_{1}^{(1)}(x)+$ $0.00002 f_{1}^{(3)}(x)$ with $m_{\alpha}=0.02, \alpha_{1} \approx 12.62992$ and $\alpha_{2} \approx 13.41656$. The Gaussian curvature is everywhere positive and has exactly two saddles, two global minima and two global maxima. It therefore satisfies the conditions stated in the abstract.

Now let us relax the condition that there should be exactly two global minima and two global maxima of the Gaussian curvature, but still demand that the Gaussian curvature should be everywhere positive. The surface given by $F_{1}(x)=f_{1}^{(1)}(x)+$ $0.001 f_{1}^{(2)}(x), m_{\alpha}=0.7, \alpha_{1} \approx 16.60291$, and $\alpha_{2} \approx 6.85556$ is such a Liouville surface. The cut locus from the point $(6,1)$ is depicted in Figure 16] The cut locus is still a straight line segment (with $x_{2}=$ const), and the conjugate locus still has four cusps. The grid size used was $3875 \times 800$. It is important to stress that we have tried many different starting points on this surface, but all gave straight cut loci and conjugate loci with four cusps.

The next surface we wish to discuss is given by $F_{1}(x)=f_{1}^{(1)}(x)+0.01 f_{1}^{(2)}(x)$, $m_{\alpha}=0.7, \alpha_{1} \approx 16.60291$, and $\alpha_{2} \approx 6.85556$. This surface has points of negative Gaussian curvature, and the Gaussian curvature has many local maxima and minima. The cut locus is depicted in Figure 17, approximated using a grid of size $3875 \times 800$. It clearly has more than two endpoints. The conjugate locus also clearly has more than four cusps. See Figure 18 for a comparison of corresponding ends of the cut loci on these two surfaces. In the former case, the cut locus has exactly two endpoints (one is shown in the figure). In the latter case, the presence of regions of negative Gaussian curvature has caused the forking of the corresponding endpoint into two endpoints. 

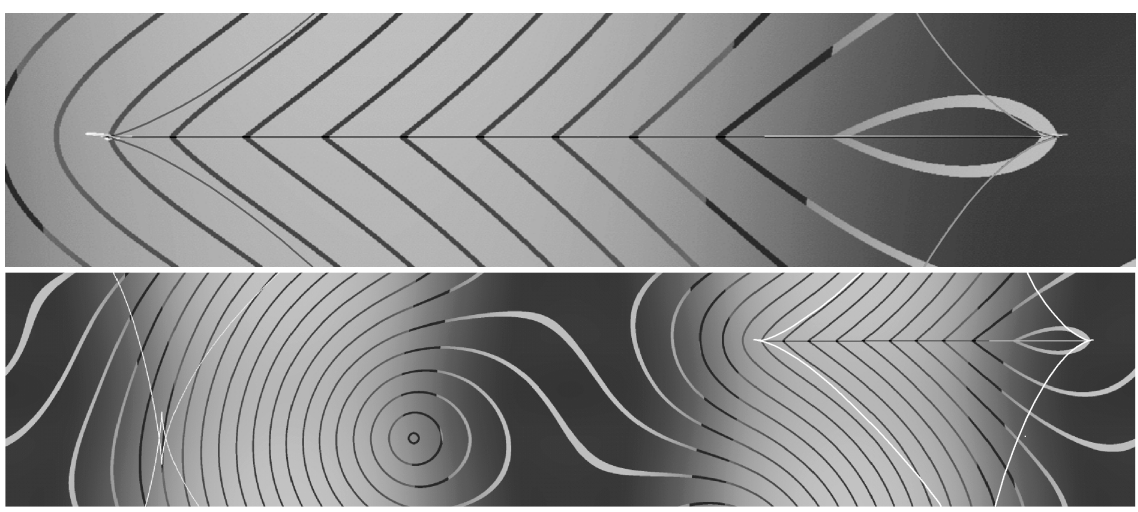

Figure 16. The cut locus from a point on a Liouville surface with many local minima and maxima of the Gaussian curvature, but which still has cut loci with two endpoints and conjugate loci with four cusps. The surface, which is given by $F_{1}(x)=f_{1}^{(1)}(x)+$ $0.001 f_{1}^{(2)}(x), m_{\alpha}=0.7, \alpha_{1} \approx 16.60291$, and $\alpha_{2} \approx 6.85556$, does not have points of negative Gaussian curvature.
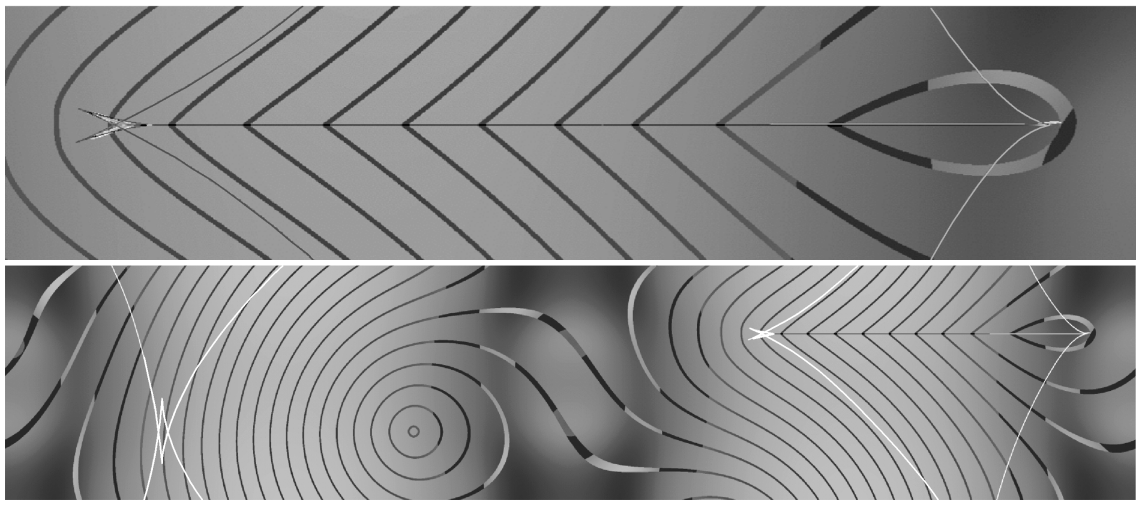

Figure 17. The cut locus from the starting point $(6,1)$ on the surface given by $F_{1}(x)=f_{1}^{(1)}(x)+0.01 f_{1}^{(2)}(x), m_{\alpha}=0.7, \alpha_{1} \approx$ 16.60291 , and $\alpha_{2} \approx 6.85556$, with a close-up of the forked cut locus.
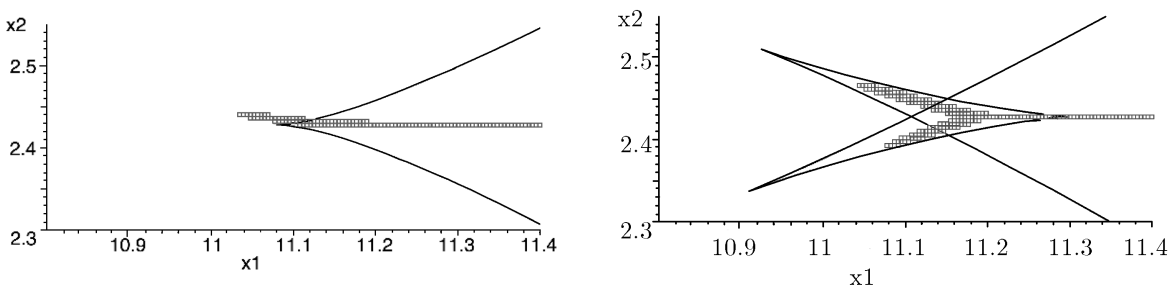

Figure 18. Close-ups of the conjugate loci from the point $(6,1)$ on the surfaces given by $F_{1}(x)=f_{1}^{(1)}(x)+0.001 f_{1}^{(2)}(x)$ and $F_{1}(x)=$ $f_{1}^{(1)}(x)+0.01 f_{1}^{(2)}(x)$ (on the left and right, respectively) with $m_{\alpha}=$ $0.7, \alpha_{1} \approx 16.60291$, and $\alpha_{2} \approx 6.85556$. The squares indicate the approximate location of the cut locus. 
These examples illustrate what we have seen throughout our investigations of Liouville surfaces of the above-mentioned type. At this stage it would appear that a surface with points of negative Gaussian curvature does not (in general) have cut loci consisting of straight line segments of the form $x_{1}=$ const or $x_{2}=$ const, neither do they (in general) have conjugate loci with exactly four cusps.

3.1. Remarks concerning how far these examples are from ellipsoids. We have of course been assuming that the examples which exhibit conjugate loci with four cusps and cut loci with exactly two endpoints are not "just" ellipsoids. If they were, then we would have been doing nothing but confirming what has already been proven in 25. The appearance of an elliptic integral of the third kind in equation (2.5), however, makes this extremely unlikely in that one specific case, since that would require a relationship between elliptic integrals of the third and lower kinds, although it is well known that one cannot reduce an elliptic integral of the third kind to an expression involving the sum of only elliptic integrals of the first and second kinds [49].

Note also that the space of ellipsoids is three dimensional (parametrized by the lengths of the axes), but we are dealing with the infinite-dimensional space generated by the $f_{1}^{(i)}$. Therefore, we can easily generate examples, which are not ellipsoids.

None of the surfaces we have constructed which have points of negative Gaussian curvature, more than six critical points of the Gaussian curvature, or critical points of Gaussian curvature which are neither global maxima, global minima, nor saddles, can be ellipsoids.

We have attempted to discover which (if any) ellipsoid the surface given by $F_{1}(x)=f_{1}^{(1)}(x)$ represents, using Section 4 of [32] as our starting point. There are many equations which can be written down, involving for example the ratio of the respective maxima of $F_{1}$ and $F_{2}$ (it is $\left(1 / \sqrt{m_{\alpha}}-1\right) / 2$ ), which can be related to a ratio of differences between squares of the lengths of the semi-axes (which is $\beta$ in 32]). All such equations must be satisfied for equivalence to hold. In practice, we have not been able to establish any equivalence, but rather find (experimentally) that the surface given by $F_{1}(x)=f_{1}^{(1)}(x)$ has properties which are incompatible with an ellipsoid.

3.2. Negative Gaussian curvature: A surface of revolution. We now refer to an example from 61] (see Figure 19). It is of a surface of revolution with regions of both positive and negative Gaussian curvature. One may choose to include surfaces of revolution in the definition of Liouville surfaces (see Exercise 19 in Section 4-2 of 62 , for example) or not [32. What we wish to demonstrate with this example is a property which does apply to Liouville surfaces which are not surfaces of revolution but "very close". This property is the disconnectedness of the conjugate locus [41] on a surface with points of negative Gaussian curvature.

One more interesting point concerning this example is that the cut locus is a subarc of a meridian. One must assume that this would translate into a straight line segment of the form $x_{1}=$ const or $x_{2}=$ const for a "close" Liouville surface. This strongly suggests that the cut locus of a Liouville surface can indeed be a straight line segment, even if the Gaussian curvature is not everywhere positive. The remaining examples we will present have been designed to test exactly this conjecture. 

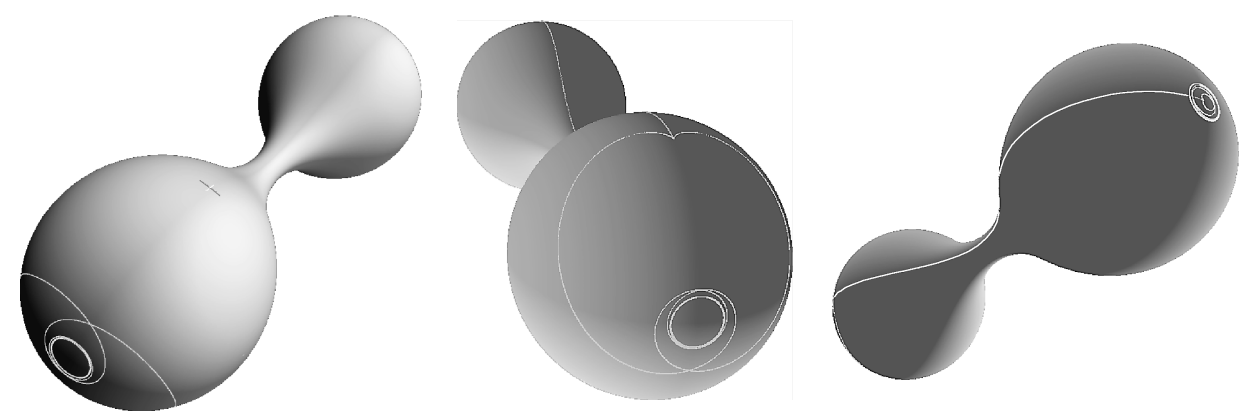

Figure 19. The cut and conjugate loci from a point on a surface of revolution with both positive and negative Gaussian curvature. The conjugate locus (the loops near the poles) is not connected. The cut locus is a single arc, a subarc of a meridian.

3.3. Alternative constructions. The Fourier-like set $\left\{f_{1}^{(i)}\right\}$ has a number of disadvantages. The least of these is a certain lack of symmetry between the sets $\left\{f_{1}^{(i)}\right\}$ and $\left\{f_{2}^{(i)}\right\}$. This asymmetry has led to the cut loci in all of our examples being horizontal $\left(x_{2}=\right.$ const $)$ rather than vertical $\left(x_{1}=\right.$ const $)$. This asymmetry can be removed to a large extent by instead using (see Section 16.3 of [1] for notation)

$$
\hat{f}_{1}^{(n)}(t)=\operatorname{sd}^{2}(n t \mid m) \quad \text { with } \quad \hat{\alpha}_{1}=4 K(m)
$$

and

$$
\hat{f}_{2}^{(n)}(t)=\operatorname{sd}^{2}(n t \mid 1-m) \quad \text { with } \quad \hat{\alpha}_{2}=4 K(1-m)
$$

where

$$
\operatorname{sd}^{2}(t \mid m)=\frac{\operatorname{sn}^{2}(t \mid m)}{\operatorname{dn}^{2}(t \mid m)}=\frac{\operatorname{sn}^{2}(t \mid m)}{1-m \operatorname{sn}^{2}(t \mid m)} .
$$

However, one practical problem remains, since any Fourier-like basis makes it difficult to control the number of critical points of the Gaussian curvature (the same problem is met by anyone wanting to approximate smooth functions with Fourier series - the more terms used, the more critical points the resultant function tends to have).

We can, however, easily construct few-parameter families of Liouville surfaces making use of the fact that the sum, difference, product, and quotient of two doubly periodic functions are also doubly periodic (Theorem 2 of Section 9 of Part II of [34]). Take, for example, the one-parameter family given by

$$
F_{1}\left(x_{1}\right)=f_{1}^{(1)}\left(x_{1}\right)+k\left[f_{1}^{(1)}\left(x_{1}\right)\right]^{2}
$$

and, recalling the relation (2.13),

$$
F_{2}\left(x_{2}\right)=f_{2}^{(1)}\left(x_{2}\right)-k\left[f_{2}^{(1)}\left(x_{2}\right)\right]^{2} .
$$

This family defines analytic compact Liouville surfaces diffeomorphic to $S^{2}$ for $-1<k<0$. 


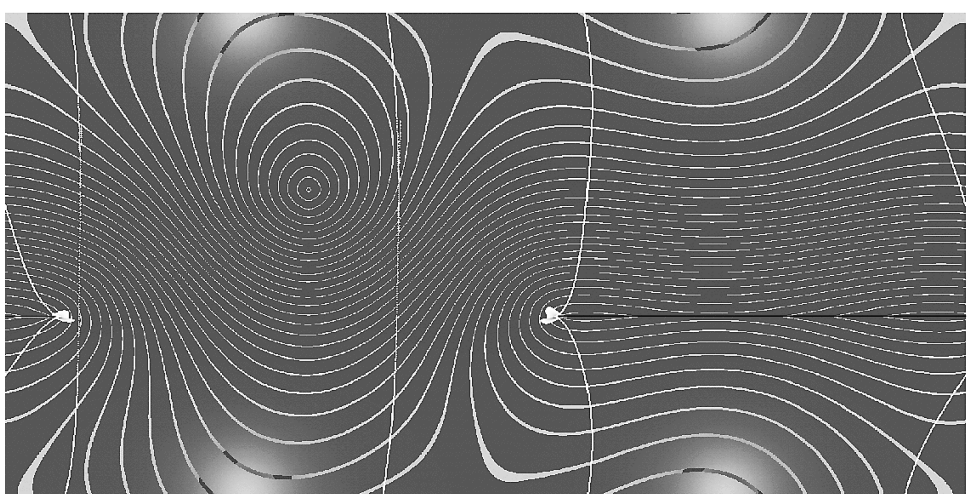

Figure 20. The cut and conjugate loci (black and white, respectively) from the point $(4,4)$ on the Liouville surface given by $F_{1}(x)=f_{1}^{(1)}(x)-0.8\left[f_{1}^{(1)}(x)\right]^{2}$ with $\alpha_{1}=\alpha_{2} \approx 12.66041$. The surface has points of both positive (dark) and negative (light) Gaussian curvature. The conjugate locus does not appear to be connected (conjugate points have been computed only up to a given distance from the starting point). The cut locus is a single straight line segment, given by $x_{2}=$ const.

What one notices is that for small negative values of $k$, up to -0.5 say, the cut loci are straight line segments of the form $x_{1}=$ const or $x_{2}=$ const and the conjugate locus has four cusps, just as we have seen in so many other examples (see Figure 151). These are surfaces with points of positive and negative curvature with exactly two saddles, two global minima, two global maxima, and no other critical points of the Gaussian curvature.

When $k$ becomes closer to -1 , the cut locus remains as before, but the conjugate locus stretches out in one direction, until two of the cusps disappear, leaving only the two cusps corresponding to the two endpoints of the cut locus. Figure 7 of [41] also illustrates this phenomenon.

We have (computationally) studied the case for which $k=-4 / 5$ and $\alpha_{1}=\alpha_{2} \approx$ 12.66041, and with the starting point $(4,4)$, in some detail. Figure 20 only includes conjugate points up to a certain distance from the starting point. We have followed the conjugate locus out to distances very far from the starting point. The conjugate locus appears to consists of two disjoint components, each becoming more vertical ( $x_{1}$ becomes more and more constant) as we consider conjugate points further and further from the starting point. Due to the boundary conditions, this asymptotic behaviour would actually present itself as a discrete set of vertical lines in Figure 20. Our experiments indicate that these vertical lines are given by $x_{1} \approx 1.05828$, $x_{1} \approx 5.27192, x_{1} \approx 7.38849$, and $x_{1} \approx 11.60213$, where the first and fourth belong to the one component of the conjugate locus, and the second and third to the other.

\section{Conclusions}

The main result of this work is that Jacobi's last geometric statement applies to a wider class of compact Liouville surfaces than just the ellipsoids. 
Every compact Liouville surface of the type $F_{1}(x)=f_{1}^{(1)}(x)+\cdots$ that we have studied which has had positive Gaussian curvature everywhere has had, from all starting points we have evaluated, a cut locus of the form $x_{2}=$ const (there is some asymmetry in our definitions of $f_{1}^{(i)}$ and $f_{2}^{(i)}$, which explains why $x_{1}=$ const has not appeared) and a conjugate locus with exactly four cusps. We hesitate to make this a conjecture, since one can easily imagine a surface of positive Gaussian curvature containing a very small region of very large Gaussian curvature, large enough to induce a conjugate point that a very similar surface without the small region of large curvature would not have.

We have therefore decided, on the basis of both our raw data and also theoretical considerations (from our experience in 20] and 61]), to make the conjecture contained in the abstract.

It does not appear to be possible to deduce the location of the cut locus by local operations on the distance map alone when the map is approximated by values on a finite-sized grid. We have found it necessary to simultaneously compute the conjugate locus, and make judgements only on the basis of both loci.

\section{REFERENCES}

1. Milton Abramowitz and Irene Stegun, Handbook of Mathematical Functions, ninth printing, Dover Publications, Inc., New York (1972).

2. Vladimir Igorevich Arnold, Topological Invariants of Plane Curves and Caustics, University Lecture Series, Volume 5, AMS, Providence RI (1994).

3. Franz Aurenhammer, Voronoi diagrams, in Jörg-Rüdiger Sack and Jorge Urrutia (editors), Handbook of computational geometry, North-Holland, Amsterdam (2000) 201-290.

4. Jean-David Benamou and Ian Solliec, An Eulerian Method for Capturing Caustics, Journal of Computational Physics, Volume 162 (2000) 132-163.

5. Jean-David Benamou, An Introduction to Eulerian Geometrical Optics (1992-2002), Journal of Scientific Computing, Volume 19 (2003) 63-93.

6. Marcel Berger, Riemannian geometry during the second half of the twentieth century, University Lecture Series, Volume 17, AMS, Providence RI (2000).

7. Marcel Berger, A Panoramic View of Riemannian Geometry, Springer-Verlag, Berlin (2003).

8. Richard L. Bishop, Decomposition of Cut Loci, Proceedings of the American Mathematical Society, Volume 65, Number 1 (1977) 133-136.

9. Harry Blum, Biological Shape and Visual Science, Journal of Theoretical Biology, Volume 38 (1973) 205-287.

10. Alexey V. Bolsinov, Vladimir S. Matveev, and Anatoly T. Fomenko, Two-dimensional Riemannian metrics with an integrable geodesic flow. Local and global geometry, Sbornik: Mathematics, Volume 189, nos. 9-10 (1998) 1441-1466.

11. Alexey V. Bolsinov and Anatoly T. Fomenko, Integrable geodesic flows on two-dimensional surfaces, Monographs in Contemporary Mathematics, Consultants Bureau, New York (2000).

12. Michael A. Buchner, The Structure of the Cut Locus in Dimension less than or equal to Six, Compositio Mathematica, Volume 37 (1978) 103-119.

13. James Damon, Smoothness and Geometry of Boundaries associated to Skeletal Structures I: Sufficient Conditions for Smoothness, Annales de l'Institut Fourier, Volume 53, Number 6 (2003) 1941-1985.

14. James Damon, Smoothness and Geometry of Boundaries associated to Skeletal Structures II: Geometry in the Blum Case, Compositio Mathematica, Volume 140, Number 6 (2004) $1657-1674$

15. Gaston Darboux, Leçons sur la Théorie Générale des Surfaces II, third edition, Chelsea Publishing Company, Bronx, New York (1972). 
16. Gaston Darboux, Leçons sur la Théorie Générale des Surfaces III, third edition, Chelsea Publishing Company, Bronx, New York (1972).

17. Jürgen Ehlers and Ezra Newman, The theory of caustics and wave front singularities with physical applications, Journal of Mathematical Physics, Volume 41, Number 6 (2000) 33443378.

18. Carl Friedrich Gauss, Disquisitiones Generales Circa Superficies Curvas, in Königliche Gesellschaft der Wissenschaften zu Göttingen, Carl Friedrich Gauss: Werke, Volume IV, Georg Olms Verlag, New York (1973) 215-258.

19. David Goldberg, What Every Computer Scientist Should Know About Floating-Point Arithmetic, ACM Computing Surveys, Volume 23, Number 1 (1991) 5-48.

20. Jens Gravesen, Steen Markvorsen, Robert Sinclair and Minoru Tanaka, The Cut Locus of a Torus of Revolution, The Asian Journal of Mathematics, Volume 9, Number 1 (2005) 103-120.

21. Karsten Grove, Critical Point Theory for Distance Functions, in Robert Greene and S.T. Yau (editors), Differential Geometry: Riemannian Geometry, Proceedings of Symposia in Pure Mathematics, Volume 54, Part 3 (1993) 357-385.

22. Max Herzberger, Modern Geometrical Optics, Pure and Applied Mathematics, Volume VIII, Interscience Publishers, New York (1958).

23. Masayuki Igarashi, Kazuyoshi Kiyohara and Kunio Sugahara, Noncompact Liouville Surfaces, Journal of the Mathematical Society of Japan, Volume 45, Number 3 (1993) 459-479.

24. Masayuki Igarashi, On compact Kähler-Liouville surfaces, Journal of the Mathematical Society of Japan, Volume 49, Number 2 (1997) 363-397.

25. Jin-Ichi Itoh and Kazuyoshi Kiyohara, The cut loci and the conjugate loci on ellipsoids, Manuscripta Mathematica, Volume 114 (2004) 247-264.

26. Jin-Ichi Itoh and Robert Sinclair, Thaw: A Tool for Approximating Cut Loci on a Triangulation of a Surface, Experimental Mathematics, Volume 13, Number 3 (2004) 309-325.

27. Carl Gustav Jakob Jacobi, Vorlesungen über Dynamik, in A. Clebsch and E. Lottner (editors), C.G.J. Jacobi's Gesammelte Werke, second edition, supplement volume, Georg Reimer, Berlin (1884).

28. Seongjai Kim, An $O(N)$ Level Set Method for Eikonal Equations, SIAM Journal on Scientific Computing, Volume 22, Number 6 (2001) 2178-2193.

29. Ron Kimmel, Arnon Amir and Alfred M. Bruckstein, Finding Shortest Paths on Surfaces Using Level Sets Propagation, IEEE Transactions on Pattern Analysis and Machine Intelligence, Volume 17, Number 6 (1995) 635-640.

30. Ron Kimmel and Nahum Kiryati, Finding Shortest Paths on Surfaces by Fast Global Approximation and Precise Local Refinement, International Journal of Pattern Recognition and Artificial Intelligence, Volume 10, Number 6 (1996) 643-656.

31. Ron Kimmel, Numerical Geometry of Images, Springer-Verlag, Berlin (2004).

32. Kazuyoshi Kiyohara, Compact Liouville Surfaces, Journal of the Mathematical Society of Japan, Volume 43, Number 3 (1991) 555-591.

33. Kazuyoshi Kiyohara, Two Classes of Riemannian Manifolds whose Geodesic Flows are Integrable, Memoirs of the American Mathematical Society, Volume 130, Number 619 (1997).

34. Konrad Knopp, Theory of Functions: Parts I and II, Dover Publications, New York (1996).

35. Richard Kunze, Franz-Erich Wolter, Thomas Rausch, Geodesic Voronoi Diagrams on Parametric Surfaces, in Computer Graphics International '97, IEEE Computer Society Press Conference Proceedings (1997) 230-237.

36. Gilles Lambare, Paulo Sergio Lucio and Andrzej Hanyga, Two-Dimensional Multivalued Traveltime and Amplitude Maps by Uniform Sampling of a Ray Field, Geophysical Journal International, Volume 125, Number 2 (1996) 584-598.

37. Tai Sing Lee, David Mumford, Richard Romeo and Victor A.F. Lamme, The Role of the Primary Visual Cortex in Higher Level Vision, Vision Research, Volume 38 (1998) 2429 2454.

38. Kung-Hao Liang, Tardi Tjahjadi and Yee-Hong Yang, Roof Edge Detection using Regularized Cubic B-Spline Fitting, Pattern Recognition, Volume 30, Number 5 (1997) 719-728.

39. D. W. Lozier and F. W. J. Olver, Numerical evaluation of special functions, in Walter Gautschi (editor), Mathematics of Computation 1943-1993: A Half-Century of Computational Mathematics, Proceedings of Symposia in Applied Mathematics, Volume 48, American Mathematical Society, Providence, Rhode Island (1994) 79-125.

An updated version is avaliable at http://math.nist.gov/nesf/ 
40. Paul Malliavin and Daniel W. Stroock, Short Time Behaviour of the Heat Kernel and its Logarithmic Derivatives, Journal of Differential Geometry, Volume 44 (1996) 550-570.

41. Christophe M. Margerin, General Conjugate Loci are not Closed, in Robert Greene and S.T. Yau (editors), Differential Geometry: Riemannian Geometry, Proceedings of Symposia in Pure Mathematics, American Mathematical Society, Volume 54, Part 3 (1993) 465-478.

42. Charles W. Misner, Kip S. Thorne and John Archibald Wheeler, Gravitation, W.H. Freeman and Company, New York (1973).

43. Sumner Byron Myers, Connections between Differential Geometry and Topology: I. Simply Connected Surfaces, Duke Mathematical Journal, Volume 1 (1935) 376-391.

44. Sumner Byron Myers, Connections between Differential Geometry and Topology: II. Closed Surfaces, Duke Mathematical Journal, Volume 2 (1936) 95-102.

45. Stanley Osher and Ronald P. Fedkiw, Level Set Methods: An Overview and Some Recent Results, Journal of Computational Physics, Volume 169 (2001) 463-502.

46. Stanley Osher, Li-Tien Cheng, Myungjoo Kang, Hyeseon Shim and Yen-Hsi Tsai, Geometric Optics in a Phase-Space-Based Level Set and Eulerian Framework, Journal of Computational Physics, Volume 179 (2002) 622-648.

47. Peter Petersen, Global Riemannian Geometry, Bulletin (New Series) of the American Mathematical Society, Volume 36, Number 3 (1999) 297-344.

48. Henri Poincaré, Sur les Lignes Géodésiques des Surfaces Convexes, Transactions of the American Mathematical Society, Volume 6 (1905) 237-274.

49. Viktor Praslov and Yuri Solovyev, Elliptic Functions and Elliptic Integrals, Translations of Mathematical Monographs, Volume 170, American Mathematical Society (1997).

50. Rajesh Ramamurthy and Rida T. Farouki, Voronoi Diagram and Medial Axis Algorithm for Planar Domains with Curved Boundaries I. Theoretical Foundations, Journal of Computational and Applied Mathematics, Volume 102 (1999) 119-141.

51. Harry Ernest Rauch, Geodesics and Curvature in Differential Geometry in the Large, Yeshiva University, Graduate School of Mathematical Sciences, G.S.M.S. - Y.U., Number 1, William Clowes and Sons, London (1959).

52. Thomas Rausch, Franz-Erich Wolter and Oliver Sniehotta, Computation of Medial Curves in Surfaces, in Tim Goodman and Ralph Martin (editors), The Mathematics of Surfaces, VII (Dundee 1996), Proceedings of the seventh IMA Mathematics of Surfaces Conference, held in Dundee, UK, September 1996, Information Geometers, Winchester, (1997) 43-68.

53. Steven J. Ruuth, Barry Merriman and Stanley Osher, A Fixed Grid Method for Capturing the Motion of Self-Intersecting Wavefronts and Related PDEs, Journal of Computational Physics, Volume 163 (2000) 1-21.

54. Takashi Sakai, Riemannian Geometry, Translations of Mathematical Monographs, Volume 149, American Mathematical Society (1996).

55. Hans Rudolf Schwarz, Numerische Mathematik, third edition, B.G. Teubner, Stuttgart (1993).

56. James A. Sethian, A Fast Marching Level Set Method for Monotonically Advancing Fronts, Proceedings of the National Academy of Sciences of the USA, Volume 93 (1996) 1591-1595.

57. James A. Sethian, Level Set Methods and Fast Marching Methods, second edition, Cambridge Monograph on Applied and Computational Mathematics, Cambridge University Press (1999).

58. Katsuhiro Shiohama, Takashi Shioya and Minoru Tanaka, The Geometry of Total Curvature on Complete Open Surfaces, Cambridge Tracts in Mathematics, Volume 159, Cambridge University Press (2003).

59. Robert Sinclair and Minoru Tanaka, Loki: Software for Computing Cut Loci, Experimental Mathematics, Volume 11, Number 1 (2002), 1-25.

60. Robert Sinclair, On The Last Geometric Statement of Jacobi, Experimental Mathematics, Volume 12, Number 4 (2003) 477-485.

61. Robert Sinclair and Minoru Tanaka, The Cut Locus of a 2-Sphere of Revolution and Toponogov's Comparison Theorem, submitted to Tohoku Mathematical Journal.

62. Dirk J. Struik, Lectures on Classical Differential Geometry, second edition, Dover Publications, New York (1988).

63. John N. Tsikilis, Efficient Algorithms for Globally Optimal Trajectories, IEEE Transactions on Automatic Control, Volume 40, Number 9 (1995) 1528-1538.

64. Franz-Erich Wolter, Distance function and cut loci on a complete Riemannian manifold, Archiv der Mathematik, Volume 32 (1979) 92-96. 
65. Franz-Erich Wolter, Cut Locus and Medial Axis in Global Shape Interrogation and Representation, MIT Design Laboratory Memorandum 92-2 and MIT Sea Grant Report (1992).

A revised version is available at

http://www.lems.brown.edu/vision/Presentations/Wolter/ReportMIT93.pdf

66. Yun Xia, Skeletonization via the Realization of the Fire Front's Propagation and Extinction in Digital Binary Shapes, IEEE Transactions on Pattern Analysis and Machine Intelligence, Volume 11, Number 10 (1989) 1076-1086.

Department of Mathematical Sciences, Faculty of Science, University of the RyuKYUS, Nishihara-Cho, OKINAWA, 903-0213 JaPAN

E-mail address: sinclair@math.u-ryukyu.ac.jp

Department of Mathematics, Tokai University, Hiratsuka City, Kanagawa, 259-1292 JAPAN

E-mail address: m-tanaka@sm.u-tokai.ac.jp 